\title{
Liouville-type results for semilinear elliptic equations in unbounded domains
}

\author{
Henri Berestycki ${ }^{a}$, François Hamel ${ }^{\text {b }}$ and Luca Rossi ${ }^{\text {a,c }}$ \\ a EHESS, CAMS, 54 Boulevard Raspail, F-75006 Paris, France \\ b Université Paul Cézanne Aix-Marseille III, LATP (UMR CNRS 6632), F.S.T. \\ Avenue Escadrille Normandie-Niemen, F-13397 Marseille Cedex 20, France \\ c Università La Sapienza Roma I, Dipartimento di Matematica, \\ Piazzale Aldo Moro 2, I-00185 Roma, Italy
}

\begin{abstract}
This paper is devoted to the study of some class of semilinear elliptic equations in the whole space:

$$
-a_{i j}(x) \partial_{i j} u(x)-q_{i}(x) \partial_{i} u(x)=f(x, u(x)), \quad x \in \mathbb{R}^{N} .
$$

The aim is to prove uniqueness of positive bounded solutions - Liouville type theorems. Along the way, we establish also various existence results.

We first derive a sufficient condition, directly expressed in terms of the coefficients of the linearized operator, which guarantees the existence result as well as the Liouville property. Then, following another approach, we establish other results relying on the sign of the principal eigenvalue of the linearized operator about $u=0$, of some limit operator at infinity which we define here. This framework will be seen to be the most general one. We also derive the large time behavior for the associated evolution equation.
\end{abstract}

\section{Introduction and main results}

In this paper, we consider the problem of existence and uniqueness of positive bounded solutions of semilinear elliptic equations in the whole space $\mathbb{R}^{N}$. Our model equation is: $-\triangle u=f(u)$. It is known that, if $\Omega$ is a bounded and smooth domain in $\mathbb{R}^{N}$, if $f$ is of class $C^{1}, f(0)=0$ and $s \mapsto f(s) / s$ is strictly decreasing, then the positive bounded solution $u$ of

$$
\begin{cases}-\triangle u=f(u), & \text { in } \Omega, \\ u=0 & \text { on } \partial \Omega\end{cases}
$$


is unique, when it exists (see e.g. [1]). This result remains true also for more general elliptic equations such as

$$
\begin{cases}-a_{i j}(x) \partial_{i j} u-q_{i}(x) \partial_{i} u-c(x) u=f(x, u), & \text { in } \Omega \\ u=0 & \text { on } \partial \Omega\end{cases}
$$

(here and in the following, we use the usual summation convention on repeated indices).

The present paper deals with extensions of this result to unbounded domains, in particular to the whole space. Therefore we study equations

$$
-a_{i j}(x) \partial_{i j} u(x)-q_{i}(x) \partial_{i} u(x)=f(x, u(x)), \quad \text { in } \mathbb{R}^{N}
$$

when $f(x, s) / s$ is decreasing in $s$. The question we address is to know wether the positive bounded solution of this equation is unique. Having in mind the characterization of bounded harmonic functions, we call such a result a non-linear Liouville type result. Note that the uniqueness result does not hold in general for unbounded solutions. As an example, consider the equation $-u^{\prime \prime}=f(u)$ in $\mathbb{R}$, with $f(s)=1-s$ for $s \geq 1$. Indeed, this equation admits infinite many positive unbounded solutions, given by $u(x)=1+c e^{ \pm x}$ for any $c>0$.

Now, clearly, the result for the bounded case does not readily extend to unbounded domains. To wit, even in one dimension it is possible to find counter-examples for the equation

$$
-u^{\prime \prime}-q u^{\prime}=f(u), \quad \text { in } \mathbb{R} .
$$

Assume that $f$ is $C^{1}$, satisfies $0<f(s) \leq f^{\prime}(0) s$ for $s \in(0,1)$ and $f(0)=f(1)=0$. Then a celebrated result of Kolmogorov, Petrovskil and Piskunov asserts that (1.2) has nontrivial heteroclinic solutions if and only if $|q| \geq 2 \sqrt{f^{\prime}(0)}$. If $q \geq 2 \sqrt{f^{\prime}(0)}$ then $u(+\infty)=$ $0, u(-\infty)=1$, while if $q \leq-2 \sqrt{f^{\prime}(0)}, u(+\infty)=1, u(-\infty)=0$ (see [12]). In these cases, since $u(x+a)$ is also a solution for all $a \in \mathbb{R}$, we see that (1.2) admits infinitely many distinct (a one-parameter family) positive bounded solutions - in addition to the constant $u \equiv 1$. Hence, the Liouville property does not hold in this case, at least for $|q| \geq 2 \sqrt{f^{\prime}(0)}$.

The goal of this paper is to understand the kind of additional conditions to be imposed on the coefficients of the operator in general equations (1.1) which yield the Liouville type property.

A first answer has been given in [3], in the homogeneous case showing that the condition on $q$ in the previous example is sharp. Indeed, it is shown there that if $f$ is of the Fisher type $\left(0<f(s) \leq f^{\prime}(0) s\right.$ for $s \in(0,1)$ and $\left.f(0)=f(1)=0\right), q$ is constant and $|q|<2 \sqrt{f^{\prime}(0)}$, then the only positive bounded solution of $-\triangle u-q \cdot \nabla u=f(u)$ in $\mathbb{R}^{N}$ is $u \equiv 1$.

In [4], the existence and uniqueness results have also been shown to hold for periodic operators in self adjoint form. A function $g(x)$ is said to be periodic in $x$ if there exist $N$ constants $l_{1}, \cdots, l_{N} \neq 0$ such that $g(x)=g\left(x+l_{i} e_{i}\right)$, for all $i=1, \cdots, N$, where $\left\{e_{1}, \cdots, e_{N}\right\}$ is the canonical basis of $\mathbb{R}^{N}$. The vector $\left(l_{1}, \cdots, l_{N}\right)$ is called period of $g$. If the functions $a_{i j}(x), f(x, s)$ are periodic in $x$, with the same period, $f(x, 0)=0$ and

$$
\begin{gathered}
\forall x \in \mathbb{R}^{N}, \quad s \mapsto f(x, s) / s \text { is decreasing in } s>0, \\
\exists M>0, \forall x \in \mathbb{R}^{N}, \forall s \geq M, \quad f(x, s) \leq 0,
\end{gathered}
$$


then the positive bounded solution of

$$
-\partial_{i}\left(a_{i j}(x) \partial_{j} u\right)=f(x, u), \quad \text { in } \mathbb{R}^{N}
$$

is unique. Furthermore, a sufficient condition for the existence of such a solution is that the periodic principal eigenvalue in $\mathbb{R}^{N}$ of the self adjoint operator $-\partial_{i}\left(a_{i j}(x) \partial_{j}\right)-f_{s}(x, 0)$ is negative (see Theorem 2.1 part 1 and Theorem 2.4 in [4]).

In the present paper, we deal with general equations - not necessarily in self adjoint form - of the type (1.1) in the whole space $\mathbb{R}^{N}$, without any periodicity assumption on the coefficients $a_{i j}, b_{i}$ and on the term $f$. Throughout the paper we always assume that $\left(a_{i j}\right)_{i, j}$ is a $N \times N$ symmetric, uniformly elliptic matrix, in the sense that there exist two positive constants $\alpha, \beta$ such that

$$
\forall x \in \mathbb{R}^{N}, \quad 0<\alpha \leq \alpha(x):=\inf _{\substack{\xi \in \mathbb{R}^{N} \\|\xi|=1}} a_{i j}(x) \xi_{i} \xi_{j} \leq \beta(x):=\sup _{\substack{\xi \in \mathbb{R}^{N} \\|\xi|=1}} a_{i j}(x) \xi_{i} \xi_{j} \leq \beta
$$

and that $q=\left(q_{1}, \cdots, q_{N}\right)$ is a $N$-dimensional vector field. The function $f(x, s): \mathbb{R}^{N} \times \mathbb{R} \rightarrow \mathbb{R}$ is always assumed to be in $C_{l o c}^{0, \gamma}\left(\mathbb{R}^{N}\right)$ with respect to the variable $x$, locally in $s$ (for some $0<\gamma<1$ ) and locally lipschitz-continuous in the variable $s$, uniformly in $x \in \mathbb{R}^{N}$, and to satisfy

$$
\forall x \in \mathbb{R}^{N}, \quad f(x, 0)=0 .
$$

We also assume that there exists $\delta>0$ such that $s \mapsto f(x, s)$ is of class $C^{1}([0, \delta])$, uniformly in $x \in \mathbb{R}^{N}$, and that

$$
a_{i j}, q_{i}, f_{s}(\cdot, 0) \in C_{l o c}^{0, \gamma}\left(\mathbb{R}^{N}\right) \cap L^{\infty}\left(\mathbb{R}^{N}\right) .
$$

In some results, we require a stronger version of condition (1.3), namely:

$$
\forall 0<s_{1}<s_{2}, \quad \inf _{x \in \mathbb{R}^{N}}\left(\frac{f\left(x, s_{1}\right)}{s_{1}}-\frac{f\left(x, s_{2}\right)}{s_{2}}\right)>0 .
$$

(Note that (1.3) and (1.5) are equivalent if $f$ is periodic.) For instance, if $f(x, s)=(\mu(x)-$ $\nu(x) s) s$, property (1.5) holds if $\inf _{\mathbb{R}^{N}} \nu>0$; if, in addition, $\sup _{\mathbb{R}^{N}} \mu<+\infty$ then (1.4) is fulfilled too.

The results in papers [3] and [4] thus point at two possible approaches to extend the Liouville property to equation (1.1). The first paper suggests to look directly at the coefficients of the linearized operator and in particular at the quantity $4 f_{s}(x, 0)-|q(x)|^{2}$. Indeed, as we have seen, if $\left(a_{i j}\right)_{i, j}$ is the identity matrix and $q$ and $f$ do not depend on $x$, the Liouville property holds if and only if that quantity is positive. The second paper shows that the principal eigenvalue of the linearized operator plays a crucial role. We follow both approaches, that we call respectively the direct and the stability approach. Thus, we derive two kind of results.

Following first the direct approach, in Section 3, we show that the Liouville property, as well as the existence result, holds if the following condition is fulfilled:

$$
\liminf _{|x| \rightarrow \infty}\left(4 \alpha(x) f_{s}(x, 0)-|q(x)|^{2}\right)>0,
$$


(we recall that $\alpha(x)$ is the smallest eigenvalue of the matrix $\left.\left(a_{i j}(x)\right)_{i, j}\right)$. More precisely, we derive:

Theorem 1.1 1) Under assumptions (1.4) and (1.6), equation (1.1) admits at least one positive bounded solution.

2) Under assumptions (1.5) and (1.6), equation (1.1) admits at most one nonnegative bounded solution, besides the trivial one: $u \equiv 0$.

Another result based on condition (1.6) is that, if the nonlinearity $f$ is of monostable type, i.e.

$$
\begin{cases}\forall s \in(0,1), & \inf _{x \in \mathbb{R}^{N}} f(x, s)>0, \\ \forall x \in \mathbb{R}^{N}, & f(x, 1)=0,\end{cases}
$$

then any super-solution $v$ of (1.1) such that $0 \leq v \leq 1$ is identically equal to 0 or to 1 . This result is a generalization of the theorem in [3], and we prove it without assuming (1.5).

Then, the stability approach is explored in Section 5. The first step is to find a quantity which plays the role played by the periodic principal eigenvalue in the periodic case. This quantity is the principal eigenvalue $\lambda_{1}(-L, \Omega)$ of an elliptic operator $-L$ in a general, possibly unbounded, domain $\Omega$, as defined in [7]. This definition reads ${ }^{1}$ :

$$
\lambda_{1}(-L, \Omega):=\sup \left\{\lambda \mid \exists \phi \in C^{2}(\Omega) \cap C^{1}(\bar{\Omega}), \phi>0 \text { and }(L+\lambda) \phi \leq 0 \text { in } \Omega\right\} .
$$

One of the simplest results for equation (1.1), relying on definition (1.8), is the following one: if (1.4) holds we show that a sufficient condition for the existence of a positive bounded solution of $(1.1)$ is that $\lambda_{1}\left(-\mathcal{L}_{0}, \mathbb{R}^{N}\right)<0$, where $-\mathcal{L}_{0}$ is the linear operator obtained by linearization around the constant solution $u=0$ of the operator appearing in (1.1), i.e.

$$
-\mathcal{L}_{0}:=-a_{i j}(x) \partial_{i j}-q_{i}(x) \partial_{i}-f_{s}(x, 0)
$$

Note that in general $-\mathcal{L}_{0}$ is not self adjoint. For special nonlinearities of the type $f(x, u)=$ $b(x) u-a(x) u^{2}$, with inf $a>0$, this existence result is due to Engländer and Pinsky (see [10], and also [9], [13]).

In one of its statement, our main result based on the stability approach (Theorem 1.3 below) requires the following uniform smoothness assumption:

$$
\left\{\begin{array}{c}
a_{i j}, q_{i} \in C^{0, \gamma}\left(\mathbb{R}^{N}\right) \cap L^{\infty}\left(\mathbb{R}^{N}\right) \\
f_{s}(\cdot, s) \in C^{0, \gamma}\left(\mathbb{R}^{N}\right) \text { uniformly in } s \in[0, \delta]
\end{array}\right.
$$

Furthermore, we require the notion of principal eigenvalue of limit operators associated with $-\mathcal{L}_{0}$, which we now define.

\footnotetext{
${ }^{1} \mathrm{By} C^{1}(\bar{\Omega})$, we mean here $C_{l o c}^{1}(\bar{\Omega})$, that is the set of functions $\phi \in C^{1}(\Omega)$ for which $\phi$ and $\nabla \phi$ can be extended by continuity on $\partial \Omega$, but which are not necessarily bounded.
} 
Definition 1.2 A limit operator (associated with $-\mathcal{L}_{0}$ ) is an operator

$$
-\mathcal{L}^{*}=-a_{i j}^{*}(x) \partial_{i j}-q_{i}^{*}(x) \partial_{i}-p^{*}(x)
$$

such that there exist a sequence $\left(x_{n}\right)_{n \in \mathbb{N}}$ in $\mathbb{R}^{N}$ and $0<\tilde{\gamma}<1$ satisfying: $\left|x_{n}\right| \rightarrow \infty$ and

$$
a_{i j}\left(x_{n}+x\right) \rightarrow a_{i j}^{*}(x), \quad q_{i}\left(x_{n}+x\right) \rightarrow q_{i}^{*}(x), \quad f_{s}\left(x_{n}+x, 0\right) \rightarrow p^{*}(x)
$$

in $C_{l o c}^{0, \tilde{\gamma}}\left(\mathbb{R}^{N}\right)$, as $n \rightarrow \infty$.

Note that $-\mathcal{L}^{*}$ is still elliptic. With this notion, we can now state our result.

Theorem 1.3 1) If (1.4) holds and there exists a limit operator $-\mathcal{L}^{*}$ such that $\lambda_{1}\left(-\mathcal{L}^{*}, \mathbb{R}^{N}\right)<$ 0 (where $\lambda_{1}$ is given by definition (1.8)), then (1.1) admits at least one positive bounded solution.

2) If (1.5),(1.9) hold and $\lambda_{1}\left(-\mathcal{L}^{*}, \mathbb{R}^{N}\right)<0$ for every limit operator $-\mathcal{L}^{*}$, then (1.1) admits at most one nonnegative bounded solution, besides the trivial one: $u \equiv 0$.

Theorem 1.3 extends the sufficient side of the results already known in the periodic self adjoint framework (Theorem 2.1 part 1) and Theorem 2.4 in [4]). In fact, in that case, the principal eigenvalue $\lambda_{1}\left(-\mathcal{L}^{*}, \mathbb{R}^{N}\right)$ of any limit operator $-\mathcal{L}^{*}$ coincides with the periodic principal eigenvalue in $\mathbb{R}^{N}$ of $-\mathcal{L}_{0}$ (see Proposition 6.7 below).

Actually, under the uniform smoothness assumption (1.9), Theorem 1.3 will be shown to contain Theorem 1.1. In fact, condition (1.9) yields the existence of limit operators, and in Section 4 we show that if (1.6) holds then the principal eigenvalue $\lambda_{1}\left(-\mathcal{L}^{*}, \mathbb{R}^{N}\right)$ of every limit operator $-\mathcal{L}^{*}$ is negative (Corollary 4.5 ). Thus, the stability approach turns out to be more general than the direct one. However, we still prove Theorem 1.1 separately. We do this for two reasons. First, the condition in Theorem 1.1, being expressed on the coefficients of the operator, is more explicit and thus can be checked more readily then the condition of the abstract result. Second, to show that Theorem 1.1 follows from an abstract approach (Theorem 1.3) requires essentially the same work as its direct derivation which we give here.

We treat also the special case when the coefficients of $\mathcal{L}_{0}$ are almost periodic, in the sense of the following definition.

Definition 1.4 A continuous function $g: \mathbb{R}^{N} \rightarrow \mathbb{R}$ is said to be almost periodic (a.p.) if from any sequence $\left(x_{n}\right)_{n \in \mathbb{N}}$ in $\mathbb{R}^{N}$ one can extract a subsequence $\left(x_{n_{k}}\right)_{k \in \mathbb{N}}$ such that $g\left(x_{n_{k}}+x\right)$ converges uniformly in $x \in \mathbb{R}^{N}$.

It is easy to see that any continuous periodic function is in particular a.p. (see e.g. [8]). We show that if the coefficients of $\mathcal{L}_{0}$ are a.p. then the principal eigenvalue of any limit operator coincides with the principal eigenvalue of $-\mathcal{L}_{0}$ and then, thanks to Theorem 1.3, we derive the following result.

Theorem 1.5 Assume that the functions $a_{i j}, q_{i}$ and $x \mapsto f_{s}(x, 0)$ are a.p. If (1.5),(1.9) hold and $\lambda_{1}\left(-\mathcal{L}_{0}, \mathbb{R}^{N}\right)<0$, then (1.1) admits at most one nonnegative bounded solution, besides the trivial one: $u \equiv 0$. 
Regarding necessary conditions, it is known that in the periodic self adjoint setting, if there exists a positive bounded solution then the periodic principal eigenvalue in $\mathbb{R}^{N}$ of the linearized operator is negative (see Theorems 2.4, part 2 in [4]). Now, we cannot proceed as for the sufficiency condition and replace the periodic principal eigenvalue with the quantity $\lambda_{1}\left(-\mathcal{L}_{0}, \mathbb{R}^{N}\right)$. In fact, the negativity of $\lambda_{1}\left(-\mathcal{L}_{0}, \mathbb{R}^{N}\right)$ is not a necessary condition for the existence result for non-self adjoint equations, even in the periodic case. As an example, consider the equation $-u^{\prime \prime}+u^{\prime}=f(u)$, where $f(0)=f(1)=0,0<f^{\prime}(0) \leq 1 / 4$ and $0<f(s) \leq f^{\prime}(0) s$ for all $s \in(0,1)$. Under these conditions, it admits positive bounded solutions ([12]), while $\lambda_{1}=1 / 4-f^{\prime}(0) \geq 0$ (see subsection 6.2). Rather, the necessary result stated in [4] is almost completely extended in Section 6 by the use of another quantity, denoted by $\lambda_{1}^{\prime}\left(-\mathcal{L}_{0}, \mathbb{R}^{N}\right)$, which differs slightly from $\lambda_{1}\left(-\mathcal{L}_{0}, \mathbb{R}^{N}\right)$. The quantity $\lambda_{1}^{\prime}$ will be seen to be a generalization of the periodic principal eigenvalue (see Proposition 6.3). The only case which is not covered is $\lambda_{1}^{\prime}=0$. What happens in that case is an open question.

To complete the picture, we only mention that in [2] a necessary and sufficient condition involving $\lambda_{1}\left(-\mathcal{L}_{0}, \mathbb{R}^{N}\right)$ is nevertheless derived for a class of non-self adjoint operators in the framework of a model arising in ecology.

Lastly, in Section 7, we make use of the existence and uniqueness results for (1.1) to fully describe the asymptotic behavior of the solution $u(x, t)$ of the evolution problem

$$
\begin{cases}\partial_{t} u-a_{i j}(x) \partial_{i j} u-q_{i}(x) \partial_{i} u=f(x, u), & t>0, x \in \mathbb{R}^{N}, \\ u(0, x)=u_{0}(x), & x \in \mathbb{R}^{N},\end{cases}
$$

with initial condition $u_{0}$.

But first, in the next section we derive a maximum principle type result which is useful in the remaining of the paper, but is also of independent interest.

\section{A strong maximum principle for strict super-solutions in unbounded domains}

Several results presented in the paper indeed rely on a refined version, for strict supersolutions, of the strong maximum principle in unbounded domains. The usual strong maximum principle states that any nonnegative super-solution $v$ of an elliptic equation cannot reach the value zero in an interior point, lest it be identically equal to zero. In other words, if $-\mathcal{L} v \geq 0$ in a domain $\Omega$ and $v$ is nonnegative and not identically equal to zero, then $v(x)>0$ for all $x \in \Omega$ (see e.g. [14]). Here, we require a stronger version which essentially says that, if $v$ is a nonnegative strong super-solution (in the sense that $-\mathcal{L} v \geq \varepsilon>0$ in $\Omega$ ) such that $\inf _{\partial \Omega} v>0$, then $\inf _{\Omega} v>0$.

We consider a generic unbounded domain $\Omega$ and define

$$
\forall x \in \Omega, \quad-\mathcal{L} u(x):=-b_{i j}(x) \partial_{i j} u(x)-p_{i}(x) \partial_{i} u(x)-c(x) u(x) .
$$

Throughout this section, we assume that $b_{i j}, p_{i}, c \in L^{\infty}(\Omega)$ and the matrix field $\left(b_{i j}\right)_{1 \leq i, j \leq N}$ is uniformly elliptic. Our strong maximum principle is proved in the following. 
Lemma 2.1 Let $\Omega$ be a general unbounded domain. Assume that there exist a positive constant $\varepsilon$ and a nonnegative function $v \in C^{2}(\Omega) \cap C(\bar{\Omega})$ such that: $-\mathcal{L} v \geq \varepsilon>0$ in $\Omega$ and, if $\Omega \neq \mathbb{R}^{N}$, $\inf _{\partial \Omega} v>0$. Then we have the following:

(i) $\inf _{\Omega} v>0$,

(ii) if $u \in C^{2}(\Omega) \cap C(\bar{\Omega})$ is such that $\sup _{\Omega} u<\infty,-\mathcal{L} u \leq 0$ and, in case $\Omega \neq \mathbb{R}^{N}, u \leq 0$ on $\partial \Omega$, then $u \leq 0$ in $\Omega$,

(iii) in case $\Omega \neq \mathbb{R}^{N}$, if $u \in C^{2}(\Omega) \cap C(\bar{\Omega})$ is such that $\sup _{\Omega} u<\infty,-\mathcal{L} u \leq 0, u \leq v$ on $\partial \Omega$, then $u \leq v$ in $\Omega$.

Proof. (i) Suppose by contradiction that $\inf _{\Omega} v=0$. Consider a nonnegative function $\theta \in C^{2}\left(\mathbb{R}^{N}\right)$ verifying:

$$
\theta(0)=0, \quad \lim _{|x| \rightarrow \infty} \theta(x)=1, \quad\|\theta\|_{C^{2}\left(\mathbb{R}^{N}\right)}<\infty .
$$

It is easy to see that there exists $\kappa>0$ sufficiently large such that

$$
\forall y \in \mathbb{R}^{N}, \quad-\mathcal{L}\left(\tau_{y} \theta\right)>-\kappa \frac{\varepsilon}{2},
$$

where we denote $\tau_{y} \theta(\cdot)=\theta(\cdot-y)$. Since $\inf _{\Omega} v=0$, we can choose $x_{0} \in \Omega$ in order to have:

$$
v\left(x_{0}\right)<\min \left\{\frac{1}{\kappa}, \inf _{\partial \Omega} v, \frac{\varepsilon}{2\|c\|_{\infty}}\right\}
$$

(treat the term $\inf _{\partial \Omega} v$ as $+\infty$ if $\Omega=\mathbb{R}^{N}$, and the same for $\varepsilon /\|c\|_{\infty}$ if $\|c\|_{\infty}=0$ ). Since $\lim _{|x| \rightarrow \infty} \theta(x)=1$, there exists a positive constant $R$ such that $\tau_{x_{0}} \theta(x) / \kappa>v\left(x_{0}\right)$ for $x \in$ $\partial B_{R}\left(x_{0}\right) \cap \bar{\Omega}$ (here and in the sequel, $B_{R}\left(x_{0}\right)$ stands for the open ball of radius $R$ and center $\left.x_{0}\right)$. Consequently, calling $\tilde{v}(x):=v(x)+\tau_{x_{0}} \theta(x) / \kappa$, one finds, for $x \in \partial B_{R}\left(x_{0}\right) \cap \bar{\Omega}$ :

$$
\tilde{v}(x)=v(x)+\tau_{x_{0}} \theta(x) / \kappa \geq \tau_{x_{0}} \theta(x) / \kappa>v\left(x_{0}\right)=\tilde{v}\left(x_{0}\right) .
$$

Hence, if

$$
\mu:=\frac{\min }{B_{R}\left(x_{0}\right)} \cap \bar{\Omega}
$$

there exists $\underline{x} \in B_{R}\left(x_{0}\right) \cap \bar{\Omega}$ such that $\tilde{v}(\underline{x})=\mu$. Actually $\underline{x}$ belongs to $B_{R}\left(x_{0}\right) \cap \Omega$, because

$$
v(\underline{x}) \leq \tilde{v}(\underline{x}) \leq \tilde{v}\left(x_{0}\right)=v\left(x_{0}\right)<\inf _{\partial \Omega} v .
$$

Moreover, $\mu \leq \tilde{v}\left(x_{0}\right)=v\left(x_{0}\right)$ and then

$$
\mu<\frac{\varepsilon}{2\|c\|_{\infty}}
$$

Finally, let $\chi$ be the $C^{2}$ function defined by $\chi(x):=\tilde{v}(x)-\mu$. We have:

$$
\chi \geq 0 \text { in } \overline{B_{R}\left(x_{0}\right)} \cap \bar{\Omega}, \quad \chi(\underline{x})=0,
$$


and by (2.1) and (2.2),

$$
\begin{aligned}
-\mathcal{L} \chi(x) & =-\mathcal{L} v(x)-\frac{1}{\kappa} \mathcal{L}\left(\tau_{x_{0}} \theta\right)(x)+c(x) \mu \\
& >\varepsilon-\frac{\varepsilon}{2}-\|c\|_{\infty} \mu \\
& >0
\end{aligned}
$$

for a.e. $x \in B_{R}\left(x_{0}\right) \cap \Omega$. Thus, the strong maximum principle yields that $\chi=0$ in the connected component of $\underline{x}$ in $\overline{B_{R}\left(x_{0}\right)} \cap \bar{\Omega}$. That is a contradiction with $-\mathcal{L} \chi>0$.

(ii) It suffices to consider the case $\sup _{\Omega} u>0$. Since $\sup _{\Omega} u<\infty$ and $\inf _{\Omega} v>0$ by (i), we have then:

$$
0<\sup _{\Omega} \frac{u}{v}<\infty
$$

Calling $w:=\left(\sup _{\Omega} u / v\right) v-u$ one easily checks that $w \geq 0$ in $\Omega$ and $\inf _{\Omega} w=0$. Furthermore

$$
-\mathcal{L} w=\left(\sup _{\Omega} \frac{u}{v}\right)(-\mathcal{L} v)+\mathcal{L} u \geq\left(\sup _{\Omega} \frac{u}{v}\right) \varepsilon>0
$$

in $\Omega$ and, in case $\Omega \neq \mathbb{R}^{N}$ one has $\inf _{\partial \Omega} w>0$. We can then apply (i) to $w$ and find a contradiction.

(iii) follows immediately from (ii) applied to the function $\tilde{u}=u-v$.

Remark 2.2 Clearly, this refined strong maximum principle does not hold if $v$ is not a strong super-solution in the sense that $-\mathcal{L} v \geq \varepsilon>0$. This can be seen on a simple example taking $\Omega=\mathbb{R}$ and $-\mathcal{L} u:=-u^{\prime \prime}+p u$, where $p$ is a positive constant. Indeed, for every $k \in \mathbb{R}$, the function $v(x):=e^{k x}$ satisfies: $-\mathcal{L} v=\left(p-k^{2}\right) v$. Thus, if $k^{2}<p$, the function $v$ is a super-solution in the sense that $-\mathcal{L} v>0$ in $\mathbb{R}$. But the fact that inf $v=0$ shows that statement (i) of Lemma 2.1 does not hold here.

The refined maximum principle (statement (i) of Lemma 2.1) plays an important role in the proof of part 2) of Theorems 1.1 and 1.3. Statement (ii) is derived from (i) and it is a maximum principle that will be used, in Section 6 , to show that $\lambda_{1}^{\prime}$ coincides with the periodic principal eigenvalue. Statement (iii) is a comparison principle without an hypothesis on the zero order term of the operator. We will use it to prove the Liouville property in the case of monostable nonlinearity, which does not necessarily fulfill the monotonicity assumption (1.3) (Theorem 3.7).

Remark 2.3 By Lemma 2.1 we infer that, if there exists a positive function $v$ satisfying $-\mathcal{L} v \geq \varepsilon>0$ in $\mathbb{R}^{N}$, the unique bounded solution of $-\mathcal{L} w=0$ in $\mathbb{R}^{N}$ is $w \equiv 0$ and that the same is true for the Dirichlet problem in a general unbounded domain, provided that $\inf _{\partial \Omega} v>0$. This is seen by applying (ii) to $w$ and $-w$. Thus, this yields uniqueness of the solution to the Dirichlet problem:

$$
\left\{\begin{array}{l}
-\mathcal{L} u=f(x), \quad u \text { bounded in } \Omega \\
\left.u\right|_{\partial \Omega}=g(x)
\end{array}\right.
$$




\section{The direct approach}

\subsection{Proof of Theorem 1.1}

Condition (1.6) is useful in that it allows one to construct a nonnegative function $\psi$, not identically equal to zero, satisfying, for every $x_{0} \in \mathbb{R}^{N} \backslash B_{R}$ with $R$ chosen sufficiently large:

$$
-a_{i j}(x) \partial_{i j}\left(\tau_{x_{0}} \psi\right)-q_{i}(x) \partial_{i}\left(\tau_{x_{0}} \psi\right) \leq f_{s}(x, 0) \tau_{x_{0}} \psi, \quad x \in \mathbb{R}^{N} .
$$

Here, $B_{R}$ denotes the open ball of radius $R$ and center 0 and $\tau_{x_{0}} \psi$ the translation by $x_{0}$ of $\psi$, i.e. $\tau_{x_{0}} \psi(\cdot):=\psi\left(\cdot-x_{0}\right)$. This fact is expressed (in a slightly more general context) in the next lemma, which is proved in the Appendix.

Lemma 3.1 Let $\mathcal{A}$ be an arbitrary subset of $\mathbb{R}^{N}$ and $-L:=-a_{i j}(x) \partial_{i j}-q_{i}(x) \partial_{i}-c(x)$ an elliptic operator satisfying:

$$
\forall x \in \mathbb{R}^{N}, \quad 0<\alpha(x):=\inf _{\substack{\xi \in \mathbb{R}^{N} \\|\xi|=1}} a_{i j}(x) \xi_{i} \xi_{j} \leq \sup _{\substack{\xi \in \mathbb{R}^{N} \\|\xi|=1}} a_{i j}(x) \xi_{i} \xi_{j} \leq \beta,
$$

where $\beta$ is a positive constant, and

$$
\sup _{x \in \mathcal{A}}|q(x)| \leq a<\infty, \quad \inf _{x \in \mathcal{A}}\left(4 \alpha(x) c(x)-|q(x)|^{2}\right)=b>0 .
$$

There exist a positive constant $r$ and a function $\psi \in C^{2}\left(\mathbb{R}^{N}\right)$, both depending only on $a, b, \beta$ and the dimension $N$, such that:

$$
\psi>0 \text { in } B_{r}, \quad \psi=0 \text { in } \mathbb{R}^{N} \backslash B_{r},
$$

and

$$
-L \tau_{x_{0}} \psi<0, \quad \text { in } B_{r}\left(x_{0}\right)
$$

for every $x_{0}$ such that $B_{r}\left(x_{0}\right) \subset \mathcal{A}$.

In the sequel, $r(a, b)$ will denote the constant $r=r(a, b, \beta, N)$ given by Lemma 3.1, where we omit its dependence on $\beta$ and $N$. The function $\psi$, as well as the next lemma, will be used to prove the existence result.

Lemma 3.2 If (1.4) holds and there exists a nonnegative bounded function $\underline{v} \in C^{2}\left(\mathbb{R}^{N}\right)$, not identically equal to zero, such that

$$
\forall x \in \mathbb{R}^{N}, \quad-a_{i j}(x) \partial_{i j} \underline{v}-q_{i}(x) \partial_{i} \underline{v} \leq f(x, \underline{v}),
$$

then there exists at least one positive bounded solution of (1.1).

Proof. Let $\underline{v}$ be a nonnegative bounded subsolution of (1.1). For $x \in \mathbb{R}^{N}$ define $\bar{v}(x):=$ $\max \left\{\|\underline{v}\|_{\infty}, M\right\}$, where $M$ is the constant given by (1.4). Then, $-a_{i j}(x) \partial_{i j} \bar{v}-q_{i}(x) \partial_{i} \bar{v}=$ $0 \geq f(x, \bar{v})$. Therefore $\underline{v}$ and $\bar{v}$ are respectively a subsolution and a super-solution of (1.1). Since $\underline{v} \leq \bar{v}$, we can apply the classic iterative method and infer the existence of a solution 
$u$ of (1.1) in $\mathbb{R}^{N}$, which satisfies: $0 \leq \underline{v} \leq u \leq \bar{v}$. We only need to show that $u>0$. Suppose that there exists $x_{0} \in \mathbb{R}^{N}$ such that $u\left(x_{0}\right)=0$. Then, using the strong maximum principle (remember that $f(x, \cdot)$ is locally lipschitz-continuous, uniformly in $x \in \mathbb{R}^{N}$ ) we find that $\underline{v} \leq u \equiv 0$, which is impossible.

Proof of Theorem 1.1 Part 1). We first show that (1.1) admits a nonnegative subsolution, not identically equal to zero. Then, we apply Lemma 3.2.

For any $\mu>0$ we have that

$$
\liminf _{|x| \rightarrow \infty}\left[4 \alpha(x)\left(f_{s}(x, 0)-\mu\right)-|q(x)|^{2}\right] \geq \liminf _{|x| \rightarrow \infty}\left(4 \alpha(x) f_{s}(x, 0)-|q(x)|^{2}\right)-4 \beta \mu .
$$

By (1.6), we know that this quantity is negative for $\mu>0$ small enough. Consider such a $\mu$ and define $c(x):=f_{s}(x, 0)-\mu$. Hence, there exists $R>0$ such that inf $|x| \geq R(4 \alpha(x) c(x)-$ $\left.|q(x)|^{2}\right)=b>0$. Set $a:=\sup _{\mathbb{R}^{N}}|q|$ and $\mathcal{A}=\mathbb{R}^{N} \backslash B_{R}$. Consider the quantity $r$ and the function $\psi$, given by Lemma 3.1, associated with the constants $a, b, \beta$ and $N$. Take $x_{0} \in \mathbb{R}^{n} \backslash B_{R+r}$. Since $B_{r}\left(x_{0}\right) \subset \mathcal{A}$ and $\tau_{x_{0}} \psi=0$ outside $B_{r}\left(x_{0}\right)$, we know that:

$$
\forall x \in \mathbb{R}^{N}, \quad-a_{i j}(x) \partial_{i j}\left(\tau_{x_{0}} \psi\right)-q_{i}(x) \partial_{i}\left(\tau_{x_{0}} \psi\right) \leq\left(f_{s}(x, 0)-\mu\right) \tau_{x_{0}} \psi
$$

Thanks to the fact that $f(x, \cdot) \in C^{1}([0, \delta])$, uniformly in $x \in \mathbb{R}^{N}$, we know that there exists $\varepsilon>0$ such that

$$
\forall x \in \mathbb{R}^{N}, \forall s \in[0, \varepsilon], \quad f(x, s) \geq\left(f_{s}(x, 0)-\mu\right) s .
$$

Therefore, the function $\underline{v}:=\varepsilon \tau_{x_{0}} \psi /\|\psi\|_{\infty}$ satisfies:

$$
\forall x \in \mathbb{R}^{N}, \quad-a_{i j}(x) \partial_{i j} \underline{v}-q_{i}(x) \partial_{i} \underline{v} \leq\left(f_{s}(x, 0)-\mu\right) \underline{v} \leq f(x, \underline{v})
$$

and we can apply Lemma 3.2.

To complete the proof of Theorem 1.1, we establish a comparison principle for sub and super-solutions of (1.1). We carry this out in a succession of three lemmas.

Lemma 3.3 Let $v \in C^{2}\left(\mathbb{R}^{N}\right)$ be a nonnegative bounded function such that

$$
\forall x \in \mathbb{R}^{N}, \quad-a_{i j}(x) \partial_{i j} v(x)-q_{i}(x) \partial_{i} v(x) \geq f(x, v(x)) .
$$

If (1.6) holds, then, either $v \equiv 0$, or $\inf _{\mathbb{R}^{N}} v>0$.

Proof. We follow the lines of the argument in [3], relying on the construction of a subsolution (which is now given by Lemma 3.1) and on the sliding method of [6].

If there exists $x_{0} \in \mathbb{R}^{N}$ such that $v\left(x_{0}\right)=0$, then the strong maximum principle yields $v \equiv 0$ (remember that $f(x, \cdot)$ is locally lipschitz-continuous) and the statement is proved.

Suppose that $v>0$ in $\mathbb{R}^{N}$. Consider the same $r$ and $\psi$ as in the proof of Theorem 1.1 part $1)$. Hence, there exist two positive constants $R$ and $\mu$ such that, for any $x_{0} \in \mathbb{R}^{N} \backslash B_{R+r}$, we have:

$$
\forall x \in B_{r}\left(x_{0}\right), \quad-a_{i j}(x) \partial_{i j} \tau_{x_{0}} \psi(x)-q_{i}(x) \partial_{i} \tau_{x_{0}} \psi(x)<\left(f_{s}(x, 0)-\mu\right) \tau_{x_{0}} \psi(x) .
$$


Moreover $\tau_{x_{0}} \psi=0$ on $\partial B_{r}\left(x_{0}\right)$. For $\varepsilon>0$ small enough, the following holds:

$$
\forall x \in \mathbb{R}^{N}, \forall s \in[0, \varepsilon], \quad f(x, s) \geq\left(f_{s}(x, 0)-\mu\right) s .
$$

Fix $y \in \mathbb{R}^{N} \backslash B_{R+r}$ and take $\varepsilon<\frac{\inf _{B_{r}(y)} v}{\|\psi\|_{\infty}}$; then define $\underline{v}:=\varepsilon \psi$. Using (3.2) and (3.3) we find, for any $x_{0} \in \mathbb{R}^{N} \backslash B_{R+r}$ and $x \in B_{r}\left(x_{0}\right)$,

$$
-a_{i j}(x) \partial_{i j} \tau_{x_{0}} \underline{v}(x)-q_{i}(x) \partial_{i} \tau_{x_{0}} \underline{v}(x)<f\left(x, \tau_{x_{0}} \underline{v}(x)\right) .
$$

We claim that

$$
\inf _{\mathbb{R}^{N} \backslash B_{R+r}} v \geq \underline{v}(0)
$$

To prove this last fact, take an arbitrary $z \in \mathbb{R}^{N} \backslash B_{R+r}$ and consider a continuous curve $\gamma:[0,1] \rightarrow \mathbb{R}^{N} \backslash B_{R+r}$ such that $\gamma(0)=y, \gamma(1)=z$. We know that $\tau_{\gamma(0)} \underline{v}<v$ in $\overline{B_{r}(\gamma(0))}$, because $\|\underline{v}\|_{\infty}=\varepsilon\|\psi\|_{\infty}<\inf _{B_{r}(y)} v$. Call

$$
t^{*}=\sup \left\{t \in[0,1], \forall s \leq t, \tau_{\gamma(s)} \underline{v} \leq v \text { in } \overline{B_{r}(\gamma(s))}\right\} .
$$

It is easily seen that $\tau_{\gamma\left(t^{*}\right) \underline{v}} \leq v$ in $\overline{B_{r}\left(\gamma\left(t^{*}\right)\right)}$. Suppose by a contradiction that $t^{*}<1$. Then, there exist then a sequence $t_{n} \searrow t^{*}$ and a sequence $\left(x_{n}\right)_{n \in \mathbb{N}}$ such that $x_{n} \in \overline{B_{r}\left(\gamma\left(t_{n}\right)\right)}$ and $\tau_{\gamma\left(t_{n}\right) \underline{v}}\left(x_{n}\right)>v\left(x_{n}\right)$. Let $\left(x_{n_{k}}\right)_{k \in \mathbb{N}}$ be a converging subsequence of $\left(x_{n}\right)$ and call $\underline{x}$ its limit. Therefore $\underline{x} \in \overline{B_{r}\left(\gamma\left(t^{*}\right)\right)}$ and

$$
\tau_{\gamma\left(t^{*}\right)} \underline{v}(\underline{x})=\lim _{k \rightarrow \infty} \tau_{\gamma\left(t_{n_{k}}\right) \underline{v}}\left(x_{n_{k}}\right) \geq \lim _{k \rightarrow \infty} v\left(x_{n_{k}}\right)=v(\underline{x}) .
$$

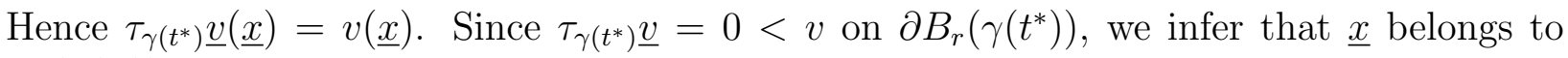
$B_{r}\left(\gamma\left(t^{*}\right)\right)$ and it is a point of local minimum for $v-\tau_{\gamma\left(t^{*}\right)}$. This implies that:

$$
\begin{aligned}
& -a_{i j}(\underline{x}) \partial_{i j} \tau_{\gamma\left(t^{*}\right) \underline{v}}(\underline{x})-q_{i}(\underline{x}) \partial_{i} \tau_{\gamma\left(t^{*}\right)} \underline{v}(\underline{x}) \geq-a_{i j}(\underline{x}) \partial_{i j} v(\underline{x})-q_{i}(\underline{x}) \partial_{i} v(\underline{x}) \\
& \geq f(\underline{x}, v(\underline{x})) \\
& =f\left(\underline{x}, \tau_{\gamma\left(t^{*}\right)} \underline{v}(\underline{x})\right) \text {. }
\end{aligned}
$$

This is in contradiction with (3.4). Thus, $t^{*}=1$ and then

$$
v(z) \geq \tau_{z} \underline{v}(z)=\underline{v}(0)=k \psi(0) .
$$

Since $\frac{\min }{B_{R+r}} v>0$, the lemma is proved.

Lemma 3.4 Let $u_{1}, u_{2} \in C\left(\mathbb{R}^{N}\right)$ be two positive bounded functions satisfying:

$$
\inf _{\mathbb{R}^{N}} u_{1}>0, \quad \inf _{\mathbb{R}^{N}}\left(u_{2}-u_{1}\right)>0 .
$$

If (1.5) holds, there exists $\varepsilon>0$ such that

$$
\forall x \in \mathbb{R}^{N}, \quad \frac{u_{2}(x)}{u_{1}(x)} f\left(x, u_{1}(x)\right) \geq f\left(x, u_{2}(x)\right)+\varepsilon .
$$


Proof. Observe first that, since $u_{2}(x)>u_{1}(x)>0$ for all $x \in \mathbb{R}^{N}$, assumption (1.5) implies that $\left(u_{2}(x) / u_{1}(x)\right) f\left(x, u_{1}(x)\right)>f\left(x, u_{2}(x)\right)$ for all $x \in \mathbb{R}^{N}$. Suppose now that there exists a sequence $\left(x_{n}\right)_{n \in \mathbb{N}}$ in $\mathbb{R}^{N}$ such that

$$
\lim _{n \rightarrow \infty}\left(\frac{f\left(x_{n}, u_{1}\left(x_{n}\right)\right)}{u_{1}\left(x_{n}\right)}-\frac{f\left(x_{n}, u_{2}\left(x_{n}\right)\right)}{u_{2}\left(x_{n}\right)}\right)=0 .
$$

Since $u_{1}$ and $u_{2}$ are bounded, there exists a subsequence of $\left(x_{n}\right)$ (that we still call $\left(x_{n}\right)$ ) such that $u_{1}\left(x_{n}\right) \rightarrow s_{1}$ and $u_{2}\left(x_{n}\right) \rightarrow s_{2}$. Clearly $0<s_{1}<s_{2} \leq\left\|u_{2}\right\|_{\infty}$. The function $s \mapsto f(x, s) / s$ is lipschitz-continuous in $\left[\inf _{\mathbb{R}^{N}} u_{1},\left\|u_{2}\right\|_{\infty}\right]$, uniformly in $x \in \mathbb{R}^{N}$, because $s \mapsto f(x, s)$ is locally lipschitz-continuous uniformly in $x$. Hence:

$$
0=\lim _{n \rightarrow \infty}\left(\frac{f\left(x_{n}, u_{1}\left(x_{n}\right)\right)}{u_{1}\left(x_{n}\right)}-\frac{f\left(x_{n}, u_{2}\left(x_{n}\right)\right)}{u_{2}\left(x_{n}\right)}\right)=\lim _{n \rightarrow \infty}\left(\frac{f\left(x_{n}, s_{1}\right)}{s_{1}}-\frac{f\left(x_{n}, s_{2}\right)}{s_{2}}\right),
$$

which is in contradiction with (1.5). Consequently, there exists $\varepsilon^{\prime}>0$ such that

$$
\forall x \in \mathbb{R}^{N}, \quad \frac{f\left(x, u_{1}(x)\right)}{u_{1}(x)}-\frac{f\left(x, u_{2}(x)\right)}{u_{2}(x)} \geq \varepsilon^{\prime},
$$

and then

$$
\frac{u_{2}(x)}{u_{1}(x)} f\left(x, u_{1}(x)\right)-f\left(x, u_{2}(x)\right) \geq \varepsilon^{\prime} u_{2}(x)>\varepsilon^{\prime} k,
$$

where $k=\inf _{\mathbb{R}^{N}} u_{2} \geq \inf _{\mathbb{R}^{N}}\left(u_{2}-u_{1}\right)>0$. Therefore, (3.5) holds with $\varepsilon=\varepsilon^{\prime} k$.

Lemma 3.5 Assume that (1.5) holds and let $u, v \in C^{2}\left(\mathbb{R}^{N}\right)$ be two nonnegative bounded functions satisfying: $\inf _{\mathbb{R}^{N}} v>0$ and

$$
-a_{i j}(x) \partial_{i j} u-q_{i}(x) \partial_{i} u \leq f(x, u), \quad-a_{i j}(x) \partial_{i j} v-q_{i}(x) \partial_{i} v \geq f(x, v) \quad \text { in } \mathbb{R}^{N} .
$$

Then $v \geq u$ in $\mathbb{R}^{N}$.

Proof. Since $\inf _{\mathbb{R}^{N}} v>0$, there exists $\kappa>0$ such that $\kappa v \geq u$ in $\mathbb{R}^{N}$. Call

$$
\kappa^{*}:=\inf \left\{\kappa>0 \mid \kappa v-u \geq 0 \text { in } \mathbb{R}^{N}\right\}
$$

and suppose that $\kappa^{*}>1$. The function $w:=\kappa^{*} v-u$ is nonnegative, of class $C^{2}$, and it satisfies: $-a_{i j} \partial_{i j} w-q_{i} \partial_{i} w \geq \kappa^{*} f(x, v)-f(x, u)$ in $\mathbb{R}^{N}$. Since

$$
\inf _{\mathbb{R}^{N}}\left(\kappa^{*} v-v\right)=\left(\kappa^{*}-1\right) \inf _{\mathbb{R}^{N}} v>0,
$$

the functions $u_{1}:=v$ and $u_{2}:=\kappa^{*} v$ satisfy the hypothesis of Lemma 3.4 and then there exists $\varepsilon>0$ such that

$$
-a_{i j} \partial_{i j} w-q_{i} \partial_{i} w \geq f\left(x, \kappa^{*} v\right)+\varepsilon-f(x, u) \quad \text { in } \mathbb{R}^{N} .
$$

Define

$$
g(x):=\left\{\begin{array}{cl}
\frac{f\left(x, \kappa^{*} v(x)\right)-f(x, u(x))}{\kappa^{*} v(x)-u(x)} & \text { if } \kappa^{*} v(x) \neq u(x) \\
0 & \text { if } \kappa^{*} v(x)=u(x) .
\end{array}\right.
$$


Since $f(x, \cdot)$ is locally lipschitz-continuous, the function $g$ belongs to $L^{\infty}\left(\mathbb{R}^{N}\right)$. Finally, calling $\mathcal{L}:=a_{i j} \partial_{i j}+q_{i} \partial_{i}+g$, we find that $-\mathcal{L} w \geq \varepsilon$ and then we can apply (i) of Lemma 2.1 to the function $w$. We infer that $\inf _{\mathbb{R}^{N}} w>0$. Thus, $h>0$ may be chosen small enough in order to have $h v(x) \leq w(x)$ for $x \in \mathbb{R}^{N}$, which contradicts the definition of $\kappa^{*}$.

By Lemmas 3.3 and 3.5, one immediately derives the following comparison theorem for sub and super-solutions of (1.1), which concludes the proof of Theorem 1.1.

Theorem 3.6 Let $u, v \in C^{2}\left(\mathbb{R}^{N}\right)$ be two nonnegative bounded functions satisfying, in $\mathbb{R}^{N}$,

$$
-a_{i j}(x) \partial_{i j} u-q_{i}(x) \partial_{i} u \leq f(x, u), \quad-a_{i j}(x) \partial_{i j} v-q_{i}(x) \partial_{i} v \geq f(x, v) .
$$

Then, under assumptions (1.5) and (1.6), either $v \equiv 0$, or $v \geq u$.

\subsection{The Liouville property for nonlinearities of monostable type}

We establish now an uniqueness result in the particular case where $u \equiv 1$ solves (1.1). This result is not contained in Theorem 3.6 because here we do not assume (1.5).

Theorem 3.7 Let $v$ be a function in $C^{2}\left(\mathbb{R}^{N}\right)$ satisfying: $0 \leq v \leq 1$ and

$$
\forall x \in \mathbb{R}^{N}, \quad-a_{i j}(x) \partial_{i j} v(x)-q_{i}(x) \partial_{i} v(x) \geq f(x, v(x)) .
$$

Assume conditions (1.6) and (1.7) hold. Then, either $v \equiv 0$ or $v \equiv 1$.

Proof. Suppose that $v$ is not identically equal to 0 and call $\mu:=\inf _{\mathbb{R}^{N}} v$. By Lemma 3.3 we know that $\mu>0$. Our goal is to show that $\mu=1$. Suppose by a contradiction that $\mu<1$. Since $\inf _{x \in \mathbb{R}^{N}} f(x, \mu)>0$ and $f$ is locally lipschitz-continuous in the second variable, $\rho$ can be chosen in order to have: $\mu<\rho<1$ and $\inf _{\mathbb{R}^{N} \times[\mu, \rho]} f>0$. Consider $x_{0} \in \mathbb{R}^{N}$ such that $v\left(x_{0}\right)<\rho$, and call $\Omega_{\rho}$ the connected component of the open set $v^{-1}((-\infty, \rho))$ containing $x_{0}$. If $\Omega_{\rho}$ were bounded one would have, by the maximum principle,

$$
v\left(x_{0}\right) \geq \min _{\overline{\Omega \rho}} v=\min _{\partial \Omega \rho} v=\rho,
$$

which is impossible. Therefore $\Omega_{\rho}$ is unbounded. Define $-\mathcal{L}=-a_{i j} \partial_{i j}-q_{i} \partial_{i}$ and $u \equiv \rho$. One finds:

$$
\begin{gathered}
-\mathcal{L} v \geq \inf _{\mathbb{R}^{N} \times[\mu, \rho]} f>0 \quad \text { and } \quad-\mathcal{L} u=0 \quad \text { in } \Omega_{\rho}, \\
\inf _{\partial \Omega_{\rho}} v>0, \quad u=v \quad \text { on } \partial \Omega_{\rho} .
\end{gathered}
$$

Thus, the hypothesis of statement (iii) in Lemma 2.1 are satisfied in $\Omega_{\rho}$. It follows then that $v \geq \rho$ in $\Omega_{\rho}$, which contradicts the fact that $v\left(x_{0}\right)<\rho$. 


\section{The principal eigenvalue of an elliptic operator in unbounded domains}

\subsection{The principal eigenvalue $\lambda_{1}$}

In this section we establish some properties of the principal eigenvalue $\lambda_{1}(-L, \Omega)$ of an elliptic operator

$$
-L=-a_{i j}(x) \partial_{i j}-q_{i}(x) \partial_{i}-c(x)
$$

in a general domain $\Omega \subset \mathbb{R}^{N}$, as defined in [7] - compare (1.8) above. The usual condition of ellipticity on the $a_{i j}$ is assumed and we further require that $a_{i j}, q_{i}, c \in C_{l o c}^{0, \gamma}(\bar{\Omega}) \cap L^{\infty}(\Omega)$.

Note that the set $E=\left\{\lambda \mid \exists \phi \in C^{2}(\Omega) \cap C^{1}(\bar{\Omega}), \phi>0,(L+\lambda) \phi \leq 0\right.$ in $\left.\Omega\right\}$ is nonempty and consequently $\lambda_{1}(-L, \Omega)$ in (1.8) is well defined. In fact, considering the function $\phi \equiv 1$, one finds $(L+\nu) \phi=(c+\nu) \phi$, for any $\nu \in \mathbb{R}$. So, if $\sup _{\Omega} c+\nu \leq 0$, then $\nu \in E$. This also shows that $\lambda_{1}(-L, \Omega) \geq-\sup _{\Omega} c \geq-\|c\|_{L^{\infty}(\Omega)}$.

If $\Omega$ is a bounded and smooth (say $C^{2, \gamma}$ ) domain of $\mathbb{R}^{N}$ then the principal eigenvalue of $-L$ in $\Omega$, with Dirichlet boundary conditions, is the unique constant $\mu \in \mathbb{R}$ such that there exists $\varphi \in C^{2, \gamma}(\bar{\Omega})$ (called principal eigenfunction), unique up to multiplication, verifying:

$$
\left\{\begin{array}{cl}
(L+\mu) \varphi=0 & \text { in } \Omega, \\
\varphi>0 & \text { in } \Omega, \\
\varphi=0 & \text { on } \partial \Omega
\end{array}\right.
$$

The following proposition states that (1.8) is indeed a characterization of the principal eigenvalue with Dirichlet boundary conditions in the case where $\Omega$ is bounded and smooth.

Proposition 4.1 [7] Let $\Omega$ be a bounded and smooth domain in $\mathbb{R}^{N}$. Then the principal eigenvalue $\mu$ given by (4.1) coincides with $\lambda_{1}(-L, \Omega)$ defined by (1.8).

The quantity $\lambda_{1}(-L, \Omega)$ is a well defined real number. We know that $\lambda_{1}(-L, \Omega)>-\infty$. It is also straightforward that $\lambda_{1}(-L, \Omega)<+\infty$. In fact observe that if $\Omega^{\prime} \subseteq \Omega^{\prime \prime}$ then $\lambda_{1}\left(-L, \Omega^{\prime}\right) \geq \lambda_{1}\left(-L, \Omega^{\prime \prime}\right)$ by definition. Consider then an arbitrary sphere $B \subset \Omega$ and call $\mu_{B}$ its classical principal eigenvalue, in the sense of (4.1) with $\Omega=B$. By Proposition 4.1 we know that $\mu_{B}=\lambda_{1}(-L, B)$. Consequently, since $B \subset \Omega$, we find that $\lambda_{1}(-L, \Omega) \leq$ $\lambda_{1}(-L, B)=\mu_{B}$.

By now, we will refer to the principal eigenvalue of an operator $-L$ in a domain $\Omega$ as the real number $\lambda_{1}(-L, \Omega)$ given by $(1.8)$.

Berestycki, Nirenberg and Varadhan showed in [7] that the principal eigenvalue in bounded domains depends continuously (in some sense) on the inclusion of the domains. Namely, if $\Omega$ is bounded and $\left(\Omega_{n}\right)_{n \in \mathbb{N}}$ is a sequence of nonempty open sets such that $\bar{\Omega}_{n} \subset \Omega_{n+1}, \cup \Omega_{n}=\Omega$, then the (decreasing) sequence of the principal eigenvalues in $\Omega_{n}$ converges to the principal eigenvalue in $\Omega$ (see the proof of Theorem 2.1 in [7]). It is natural to ask whether the same kind of continuity holds for unbounded domains. The following result answers affirmatively this question. 
Proposition 4.2 Let $\Omega$ be a general domain in $\mathbb{R}^{N}$ and $\left(\Omega_{n}\right)_{n \in \mathbb{N}}$ be a sequence of nonempty open sets such that

$$
\Omega_{n} \subset \Omega_{n+1}, \quad \bigcup_{n \in \mathbb{N}} \Omega_{n}=\Omega
$$

Then $\lambda_{1}\left(-L, \Omega_{n}\right) \searrow \lambda_{1}(-L, \Omega)$ as $n \rightarrow+\infty$.

Proof. This is a slightly more general statement than in [7], in that the $\Omega_{n}$ are not assumed to be bounded, and we do not assume here that $\bar{\Omega}_{n} \subset \Omega_{n+1}$. However, the proof is just the demonstration that the method in [7] also applies to this case. For the sake of completeness we include it here.

As emphasized above, the principal eigenvalue in different domains (associated with the same operator $-L)$ is non-increasing with respect to the inclusion of the domains. For short, call $\lambda_{1, n}:=\lambda_{1}\left(-L, \Omega_{n}\right)$ and $\lambda_{1}:=\lambda_{1}(-L, \Omega)$. Hence the sequence $\left(\lambda_{1, n}\right)_{n \in \mathbb{N}}$ is non-increasing and then

$$
\lambda_{1} \leq \lim _{n \rightarrow \infty} \lambda_{1, n} .
$$

It is convenient to deal with bounded and smooth domains instead of the $\Omega_{n}$. So consider a family of bounded and smooth domains $\tilde{\Omega}_{n}$ such that

$$
\tilde{\Omega}_{n} \subset \Omega_{n}, \quad \overline{\tilde{\Omega}_{n}} \subset \tilde{\Omega}_{n+1}, \quad \bigcup_{n \in \mathbb{N}} \tilde{\Omega}_{n}=\Omega,
$$

and call $\tilde{\lambda}_{n}:=\lambda_{1}\left(-L, \tilde{\Omega}_{n}\right)$. Always by monotonicity, we have that

$$
\lambda_{1} \leq \lim _{n \rightarrow \infty} \lambda_{1, n} \leq \lim _{n \rightarrow \infty} \tilde{\lambda}_{n}
$$

so it is sufficient to show that $\tilde{\lambda}:=\lim _{n \rightarrow+\infty} \tilde{\lambda}_{n} \leq \lambda_{1}$. To achieve this goal, choose a point $x_{0} \in \tilde{\Omega}_{0}$ and consider the sequence $\left(\tilde{\varphi}_{n}\right)_{n \in \mathbb{N}}$ of the principal eigenfunctions of $-L$ in $\tilde{\Omega}_{n}$, with Dirichlet boundary conditions, normalized by: $\tilde{\varphi}_{n}\left(x_{0}\right)=1$. Namely the $\tilde{\varphi}_{n}$ are positive in $\tilde{\Omega}_{n}$, vanish on $\partial \tilde{\Omega}_{n}$ and satisfy $\left(L+\tilde{\lambda}_{n}\right) \tilde{\varphi}_{n}=0$ in $\tilde{\Omega}_{n}$. Since the sequence $\left(\tilde{\lambda}_{n}\right)_{n \in \mathbb{N}}$ is bounded, using the Krylov-Safonov Harnack inequality we can find a positive constant $C(m)$, for any $m \in \mathbb{N}$, such that

$$
\forall n>m, \quad \sup _{\tilde{\Omega}_{m}} \tilde{\varphi}_{n} \leq C(m) \inf _{\tilde{\Omega}_{m}} \tilde{\varphi}_{n} \leq C(m)
$$

(see for instance [11]). Thus, by standard interior estimates, we know that there exists a subsequence of $\left(\tilde{\varphi}_{n}\right)_{n>m}$ which converges in $C^{2, \tilde{\gamma}}\left(\tilde{\Omega}_{m-1}\right)$, for some $\tilde{\gamma} \in[0, \gamma)$, to a function $\varphi_{\infty}$ satisfying

$$
(L+\tilde{\lambda}) \varphi_{\infty}=0 \quad \text { in } \tilde{\Omega}_{m-1} .
$$

Finally, using a diagonal extraction method, we can find a particular sub-sequence of $\left(\tilde{\varphi}_{n}\right)_{n \in \mathbb{N}}$ converging to $\varphi_{\infty}$ in $C_{l o c}^{2, \tilde{\gamma}}(\Omega)$. Furthermore $\varphi_{\infty}\left(x_{0}\right)=1, \varphi_{\infty} \geq 0$ and $(L+\tilde{\lambda}) \varphi_{\infty}=0$ in $\Omega$ and then the strong maximum principle yields $\varphi_{\infty}>0$ in $\Omega$. This means that $\varphi_{\infty}$ can be taken as a test super-solution in (1.8) and then $\lambda_{1} \geq \tilde{\lambda}$.

In the sequel we denote by $\varphi_{\infty}$ a positive solution of

$$
\left(L+\lambda_{1}\right) \varphi_{\infty}=0 \quad \text { in } \mathbb{R}^{N},
$$


and we call it a generalized principal eigenfunction in $\mathbb{R}^{N}$ of the operator $-L$. Such a function always exists, as shown in the proof of Proposition 4.2, but the function constructed there depends a priori on the choice of the sequence $\left(\tilde{\Omega}_{n}\right)_{n \in \mathbb{N}}$ covering $\mathbb{R}^{N}$ and on the extracted subsequence. Furthermore it may not be in $L^{\infty}(\Omega)$ because the sequence $C(m)$ may be divergent. For instance, for the operator $L u=u^{\prime \prime}-u^{\prime}$ in $\mathbb{R}$, if we choose $\Omega_{n}=(-n, n)$ and $x_{0}=0$, then a simple calculation shows that

$$
\phi_{\infty}(x)=\lim _{n \rightarrow \infty} \cos \left(\frac{\pi}{2 n} x\right) e^{x / 2}=e^{x / 2}
$$

So, to prove Proposition 4.2, it is essential that we allow in (1.8) the super-solutions $\phi$ to be unbounded. Otherwise, as we will se later, in general one would obtain a different value for $\lambda_{1}$.

\subsection{Upper bounds for the principal eigenvalue in terms of the coefficients}

An application of Lemma 3.1 concerns the sign of the principal eigenvalue of $-L=$ $-a_{i j}(x) \partial_{i j}-q_{i}(x) \partial_{i}-c(x)$ in large domains. We always assume that the $a_{i j}$ satisfy the usual elliptic condition:

$$
0<\alpha \leq \alpha(x):=\inf _{|\xi|=1} a_{i j}(x) \xi_{i} \xi_{j} \leq \beta(x):=\sup _{|\xi|=1} a_{i j}(x) \xi_{i} \xi_{j} \leq \beta,
$$

for $x \in \Omega$, and that $a_{i j}, q_{i}, c \in C_{l o c}^{0, \gamma}(\bar{\Omega}) \cap L^{\infty}(\Omega)$. Furthermore, for any $a, b>0$, we denote by $r(a, b)$ the positive constant given by Lemma 3.1 .

Theorem 4.3 Let $\Omega$ be a general domain in $\mathbb{R}^{N}$. Suppose that there exist $x_{0} \in \Omega$ and two positive constants $a, b$ such that:

$$
\begin{gathered}
B_{r(a, b)}\left(x_{0}\right) \subset \Omega, \\
\forall x \in B_{r(a, b)}\left(x_{0}\right), \quad|q(x)| \leq a \quad \text { and } \quad 4 \alpha(x) c(x)-|q(x)|^{2} \geq b .
\end{gathered}
$$

Then $\lambda_{1}(-L, \Omega)<0$.

Proof. Let us call $r$ the constant $r(a, b)$. Consider the function $\psi$ given by Lemma 3.1, associated with the constants $a, b, \beta$ and $N$. Call $\mu$ and $\varphi$ respectively the principal eigenvalue and eigenfunction of $-L$ in $B_{r}\left(x_{0}\right)$. That is, $\mu$ and $\varphi$ satisfy (4.1) with $\Omega=B_{r}\left(x_{0}\right)$. We will show that $\mu<0$. The proof is similar to the one of Proposition 4.1 (see [7]). Using the Hopf lemma, applied to the function $\varphi$, one can find a positive number $k$ such that $k \varphi \geq \tau_{x_{0}} \psi$ in $\overline{B_{r}\left(x_{0}\right)}$. Call $k^{*}=\inf \left\{k>0 \mid k \varphi \geq \tau_{x_{0}} \psi\right.$ in $\left.\overline{B_{r}\left(x_{0}\right)}\right\}$, and $w:=k^{*} \varphi-\tau_{x_{0}} \psi$. Obviously $k^{*} \geq 0$ and $w \geq 0$ in $\overline{B_{r}\left(x_{0}\right)}$. By Lemma 3.1 we have:

$$
\forall x \in B_{r}\left(x_{0}\right), \quad-L w(x)=-k^{*} L \varphi(x)+L\left(\tau_{x_{0}} \psi\right)(x)>k^{*} \mu \varphi(x) .
$$

We argue by contradiction and assume that $\mu \geq 0$. Then we have: $-L w>0$ in $B_{r}\left(x_{0}\right)$. If there exists $\underline{x} \in B_{r}\left(x_{0}\right)$ such that $w(\underline{x})=0$ then the strong maximum principle yields $w \equiv 0$, that is in contradiction with the fact that $-L w>0$. Therefore $w$ must be strictly positive 
in $B_{r}\left(x_{0}\right)$ and then, using again the Hopf's lemma, it is possible to find $\varepsilon>0$ such that $\varepsilon \varphi \leq w$ in $\overline{B_{r}\left(x_{0}\right)}$. This is in contradiction with the definition of $k^{*}$. Thus, $\mu<0$ and then, from Proposition 4.1, we infer that $\lambda_{1}(-L, \Omega)<0$.

Theorem 4.4 Assume that either $\Omega=\mathbb{R}^{N}$, or $\Omega$ is a general domain satisfying

$$
\sup _{x \in \Omega} \operatorname{dist}(x, \partial \Omega)=\infty .
$$

Call

$$
m:=\liminf _{\substack{x \in \Omega \\|x| \rightarrow \infty}}\left(4 \alpha(x) c(x)-|q(x)|^{2}\right) .
$$

Then we have the following estimates on the principal eigenvalue:

$$
\lambda_{1}(-L, \Omega) \leq \begin{cases}-\frac{m}{4 \liminf _{\substack{x \in \Omega \\|x| \rightarrow \infty}} \alpha(x)} \quad \text { if } m \leq 0, \\ -\frac{m}{4 \limsup _{\substack{x \in \Omega \\|x| \rightarrow \infty}} \alpha(x)} \quad \text { if } m>0,\end{cases}
$$

and also

$$
\lambda_{1}(-L, \Omega) \leq-\frac{1}{4 \alpha} \liminf _{\substack{x \in \Omega \\|x| \rightarrow \infty}}\left(4 \alpha c(x)-|q(x)|^{2}\right) .
$$

Proof. Take an arbitrary $\mu$ such that:

$$
\mu>-\frac{m}{4 \liminf _{\substack{x \in \Omega \\|x| \rightarrow \infty}} \alpha(x)} \quad \text { if } m \leq 0,
$$

or

$$
-\frac{m}{4 \limsup _{\substack{x \in \Omega \\|x| \rightarrow \infty}} \alpha(x)}<\mu<0 \quad \text { if } m>0 .
$$

To prove (4.2) it suffices to show that $\lambda_{1}(-L, \Omega)<\mu$. By the choice of $\mu$ we know that

$$
\begin{aligned}
\liminf _{\substack{x \in \Omega \\
|x| \rightarrow \infty}}\left[4 \alpha(x)(\mu+c(x))-|q(x)|^{2}\right] & \geq 4 \liminf _{\substack{x \in \Omega \\
|x| \rightarrow \infty}}(\alpha(x) \mu)+m \\
& >0 .
\end{aligned}
$$

Hence $R>0$ may be chosen large enough in order to have:

$$
b:=\inf _{\substack{x \in \Omega \\|x| \geq R}}\left[4 \alpha(x)(\mu+c(x))-|q(x)|^{2}\right]>0 .
$$

Call $a:=\sup _{\Omega}|q|$ and $r:=r(a, b)$. By the hypothesis on $\Omega$ we know that there exists $x_{1} \in \Omega$ such that $B_{R+2 r}\left(x_{1}\right) \subset \Omega$. If $x_{1}=0$ take an arbitrary point $x_{0}$ on $\partial B_{R+r}$, otherwise set $x_{0}:=x_{1}+(R+r) x_{1} /\left|x_{1}\right|$. In each case we have that $B_{r}\left(x_{0}\right) \subset B_{R+2 r}\left(x_{1}\right) \subset \Omega$ and $B_{r}\left(x_{0}\right) \subset \mathbb{R}^{N} \backslash B_{R}$. Thus, $4 \alpha(x)(\mu+c(x))-|q(x)|^{2} \geq b$ for all $x \in B_{r}\left(x_{0}\right)$. Consequently, 
we can apply Theorem 4.3 to the operator $-(L+\mu)$ and infer that $\lambda_{1}(-(L+\mu), \Omega)<0$, namely

$$
\sup \left\{\lambda \mid \exists \phi \in C^{2}(\Omega) \cap C^{1}(\bar{\Omega}), \phi>0 \text { and }(L+\mu+\lambda) \phi \leq 0 \text { in } \Omega\right\}<0 .
$$

Since

$$
\begin{aligned}
\lambda_{1}(-(L+\mu), \Omega) & =\sup \left\{\lambda \mid \exists \phi \in C^{2}(\Omega) \cap C^{1}(\bar{\Omega}), \phi>0,(L+\mu+\lambda) \phi \leq 0\right\} \\
& =\sup \left\{\lambda-\mu \mid \exists \phi \in C^{2}(\Omega) \cap C^{1}(\bar{\Omega}), \phi>0,(L+\lambda) \phi \leq 0\right\} \\
& =\lambda_{1}(-L, \Omega)-\mu,
\end{aligned}
$$

we conclude that $\lambda_{1}(-L, \Omega)<\mu$.

To prove (4.3), take

$$
\mu>-\frac{1}{4 \alpha} \liminf _{\substack{x \in \Omega \\|x| \rightarrow \infty}}\left(4 \alpha c(x)-|q(x)|^{2}\right) .
$$

Since

$$
\liminf _{\substack{x \in \Omega \\|x| \rightarrow \infty}}\left[4 \alpha(\mu+c(x))-|q(x)|^{2}\right]>0
$$

then we have that

$$
\liminf _{\substack{x \in \Omega \\|x| \rightarrow \infty}}\left[4 \alpha(x)(\mu+c(x))-|q(x)|^{2}\right]>0 .
$$

Proceeding as above we find that $\lambda_{1}(-L, \Omega)<\mu$.

We apply now this result to the linearized operator $-\mathcal{L}_{0}=-a_{i j}(x) \partial_{i j}-q_{i}(x) \partial_{i}-f_{s}(x, 0)$ and to its limit operators, defined by Definition 1.2.

Corollary 4.5 If (1.6) holds, then $\lambda_{1}\left(-\mathcal{L}_{0}, \mathbb{R}^{N}\right)<0$ and, for any limit operator $-\mathcal{L}^{*}$, $\lambda_{1}\left(-\mathcal{L}^{*}, \mathbb{R}^{N}\right)<0$.

Proof. Assume that

$$
m:=\liminf _{|x| \rightarrow \infty}\left(4 \alpha(x) f_{s}(x, 0)-|q(x)|^{2}\right)>0 .
$$

Then, from estimate (4.2) it follows immediately that $\lambda_{1}\left(-\mathcal{L}_{0}, \mathbb{R}^{N}\right)<0$. Now, let $-\mathcal{L}^{*}=$ $-a_{i j}^{*}(x) \partial_{i j}-q_{i}^{*}(x) \partial_{i}-p^{*}(x)$ be a limit operator associated with $-\mathcal{L}_{0}$. By definition, there exists a sequence $\left(x_{n}\right)_{n \in \mathbb{N}}$ in $\mathbb{R}^{N}$ such that $\left|x_{n}\right| \rightarrow \infty$ and

$$
\forall x \in \mathbb{R}^{N}, \quad a_{i j}\left(x_{n}+x\right) \rightarrow a_{i j}^{*}(x), \quad q_{i}\left(x_{n}+x\right) \rightarrow q_{i}^{*}(x), \quad f_{s}\left(x_{n}+x, 0\right) \rightarrow p^{*}(x) .
$$

For $x \in \mathbb{R}^{N}$, call $\alpha^{*}(x)$ the smallest eigenvalue of the matrix $\left(a_{i j}^{*}(x)\right)_{i, j}$. It is easy to check that $\alpha^{*}(x)=\lim _{n \rightarrow \infty} \alpha\left(x_{n}+x\right)$. Hence,

$$
\forall x \in \mathbb{R}^{N}, \quad 4 \alpha^{*}(x) p^{*}(x)-\left|q^{*}(x)\right|^{2}=\lim _{n \rightarrow \infty}\left(4 \alpha\left(x_{n}+x\right) f_{s}\left(x_{n}+x, 0\right)-\left|q\left(x_{n}+x\right)\right|^{2}\right) \geq m .
$$

Therefore, applying Theorem 4.4 to the operator $-\mathcal{L}^{*}$, we infer that $\lambda_{1}\left(-\mathcal{L}^{*}, \mathbb{R}^{N}\right)<0$.

Remark 4.6 Condition (1.6) is far from being a necessary condition to have $\lambda_{1}\left(-\mathcal{L}_{0}, \mathbb{R}^{N}\right)<$ 0 (even in the periodic case). This is illustrated by the by next example. Consider the operator $-\mathcal{L} u=-u^{\prime \prime}+q(x) u^{\prime}-u$ in $\mathbb{R}$, with $q(x)=0$ in $[0,2 \pi], q(3 \pi)=3, q(4 \pi)=0, q$ otherwise arbitrary in $(2 \pi, 4 \pi)$, and then extended by periodicity with period $4 \pi$. One can easily check that $\lambda_{1}(-\mathcal{L}, \mathbb{R}) \leq \lambda_{1}(-\mathcal{L},(0,2 \pi))=-3 / 4$, but $\liminf _{|x| \rightarrow \infty}\left(4-q^{2}(x)\right) \leq-5$. 


\section{The stability approach}

\subsection{The existence result}

We start with the proof of Theorem 1.3 part 1). As usual, we call

$$
-\mathcal{L}_{0}:=-a_{i j}(x) \partial_{i j}-q_{i}(x) \partial_{i}-f_{s}(x, 0)
$$

Theorem 5.1 If (1.4) holds and $\lambda_{1}\left(-\mathcal{L}_{0}, \mathbb{R}^{N}\right)<0$, then there exists at least one positive bounded solution of (1.1).

Proof. Since $\lambda_{1}\left(-\mathcal{L}_{0}, \mathbb{R}^{N}\right)<0$, it follows from Proposition 4.2 that there exists $R>0$ such that $\lambda_{R}:=\lambda_{1}\left(-\mathcal{L}_{0}, B_{R}\right)<0$. Call $\varphi_{R}$ the associated principal eigenfunction, namely (4.1) holds with $L=\mathcal{L}_{0}, \varphi=\varphi_{R}, \mu=\lambda_{R}$ and $\Omega=B_{R}$ (compare Proposition 4.1). Since $f(x, \cdot) \in C^{1}([0, \delta])$, uniformly in $x \in \mathbb{R}^{N}$, there exists $\varepsilon>0$ such that

$$
\forall x \in \mathbb{R}^{N}, \forall s \in[0, \varepsilon], \quad f(x, s) \geq\left(f_{s}(x, 0)+\lambda_{R}\right) s .
$$

We take $\varepsilon<M$, where $M$ is the constant given by (1.4), and define

$$
\underline{v}(x):= \begin{cases}\frac{\varepsilon}{\left\|\varphi_{R}\right\|_{\infty}} \varphi_{R}(x) & \text { if } x \in B_{R}, \\ 0 & \text { if } x \in \mathbb{R}^{N} \backslash B_{R} .\end{cases}
$$

For $|x|>R$ we have that $-a_{i j}(x) \partial_{i j} \underline{v}-q_{i}(x) \partial_{i} \underline{v}=f(x, \underline{v})$. For $|x|<R$ by (5.1) we have:

$$
-a_{i j}(x) \partial_{i j} \underline{v}-q_{i}(x) \partial_{i} \underline{v}=\left(f_{s}(x, 0)+\lambda_{R}\right) \underline{v} \leq f(x, \underline{v}) .
$$

Hence, $\underline{v}$ is a generalized subsolution of (1.1), in the sense that it is the connection of two subsolutions (see [5]); furthermore, it is continuous in $\mathbb{R}^{N}$. Now set $\bar{v}(x):=M$, for $x \in \mathbb{R}^{N}$. By (1.4) we have that $-a_{i j}(x) \partial_{i j} \bar{v}-q_{i}(x) \partial_{i} \bar{v} \geq f(x, \bar{v})$. Therefore $\underline{v}$ and $\bar{v}$ are respectively a subsolution and a super-solution of (1.1). Since $\underline{v} \leq \bar{v}$, we can argue as in the proof of Lemma 3.2 and infer the existence of a solution $u$ of $(1.1)$ in $\mathbb{R}^{N}$ which satisfies: $0 \leq \underline{v} \leq u \leq \bar{v}$. Using the strong maximum principle (remember that $f(x, \cdot)$ is locally lipschitz-continuous, uniformly in $x \in \mathbb{R}^{N}$ ) we find that $u>0$.

Remark 5.2 In the proof of Theorem 5.1, we do not really use assumption (1.4), but only the fact that

$$
\exists M>0, \quad \forall x \in \mathbb{R}^{N}, \quad f(x, M) \leq 0 .
$$

In fact, part 1) of Theorem 1.3 (and also of Theorem 1.1) holds under this weaker assumption. This is not the case of Theorem 7.1 below, which makes essentially use of (1.4).

The proof of Theorem 1.3 part 1) is completed by the following result:

Proposition 5.3 Let $-\mathcal{L}^{*}$ be a limit operator in the sense of Definition 1.2. Then $\lambda_{1}\left(-\mathcal{L}^{*}, \mathbb{R}^{N}\right) \geq \lambda_{1}\left(-\mathcal{L}_{0}, \mathbb{R}^{N}\right)$. 
Proof. For simplicity set $\lambda_{1}:=\lambda_{1}\left(-\mathcal{L}_{0}, \mathbb{R}^{N}\right)$. By Definition 1.2, there exists a sequence $\left(x_{n}\right)_{n \in \mathbb{N}}$ in $\mathbb{R}^{N}$ and a real number $0<\tilde{\gamma}<1$ such that: $\left|x_{n}\right| \rightarrow \infty$,

$$
a_{i j}\left(x_{n}+x\right) \rightarrow a_{i j}^{*}(x), \quad q_{i}\left(x_{n}+x\right) \rightarrow q_{i}^{*}(x), \quad f_{s}\left(x_{n}+x, 0\right) \rightarrow p^{*}(x)
$$

in $C_{l o c}^{0, \tilde{\gamma}}\left(\mathbb{R}^{N}\right)$ and $-\mathcal{L}^{*}=-a_{i j}^{*} \partial_{i j}-q_{i}^{*} \partial_{i}-p^{*}$. Let $\varphi_{\infty}$ be a generalized principal eigenfunction of $-\mathcal{L}_{0}$ in $\mathbb{R}^{N}$. Namely $\varphi_{\infty}>0$ and $\left(\mathcal{L}_{0}+\lambda_{1}\right) \varphi_{\infty}=0$ in $\mathbb{R}^{N}$ (compare the proof of Proposition 4.2 above). Define

$$
\varphi_{n}(x):=\frac{\varphi_{\infty}\left(x_{n}+x\right)}{\varphi_{\infty}\left(x_{n}\right)} .
$$

For $x \in \mathbb{R}^{N}$ a translation of the equation by $x_{n}$ yields:

$$
-a_{i j}\left(x_{n}+x\right) \partial_{i j} \varphi_{n}(x)-q_{i}\left(x_{n}+x\right) \partial_{i} \varphi_{n}(x)-f_{s}\left(x_{n}+x, 0\right) \varphi_{n}(x)=\lambda_{1} \varphi_{n}(x) .
$$

By Harnack inequality, we know that, for any fixed $R>0$, there exists a constant $C(R)>0$ such that

$$
\left\|\varphi_{n}\right\|_{L^{\infty}\left(B_{R+1}\right)} \leq C(R) \inf _{B_{R+1}} \varphi_{n} \leq C(R),
$$

where the last inequality holds because $\varphi_{n}(0)=1$. Hence, using standard interior estimates, we may extract a subsequence of $\varphi_{n}$ which converges in $C^{2, \tilde{\gamma}}\left(\overline{B_{R}}\right)$. Since it is true for every $R>0$, using a diagonal method we can find a common subsequence of $\varphi_{n}$ converging in $C_{l o c}^{2, \tilde{\gamma}}\left(\mathbb{R}^{N}\right)$ to a certain nonnegative function $\varphi^{*}$. It turns out that

$$
-a_{i j}^{*} \partial_{i j} \varphi^{*}-q_{i}^{*} \partial_{i} \varphi^{*}-p^{*} \varphi^{*}=\lambda_{1} \varphi^{*}, \quad \text { in } \mathbb{R}^{N},
$$

and then, the classical strong maximum principle and the fact that $\varphi^{*}(0)=1$ imply that $\varphi^{*}>0$. It follows that $\lambda_{1}\left(-\mathcal{L}^{*}, \mathbb{R}^{N}\right) \geq \lambda_{1}$.

Remark 5.4 Without assumption (1.4) this existence result (Theorem 5.1) may fail to hold. Consider the equation $-\triangle u=f(u)$ in $\mathbb{R}^{N}$, where $f$ is such that $f^{\prime}(0)>0$ and $\inf _{[s,+\infty)} f>0$ for all $s>0$. It is easy to see that $\lambda_{1}\left(-\mathcal{L}_{0}, \mathbb{R}^{N}\right)=-f^{\prime}(0)<0$, where $-\mathcal{L}_{0}=-\triangle-f^{\prime}(0)$. Moreover any limit operator $-\mathcal{L}^{*}$ coincides with $-\triangle-f^{\prime}(0)$ and then its principal eigenvalue in $\mathbb{R}^{N}$ is negative. Suppose that there exists a nonnegative function $v$, not identically equal to 0 , such that $-\triangle v \geq f(v)$ in $\mathbb{R}^{N}$. The strong maximum principle yields: $v>0$ in $\mathbb{R}^{N}$. For $R$ large enough, we have $\lambda_{1}\left(-\mathcal{L}_{0}, B_{R}\right)<0$. Consider the associated principal eigenfunction $\varphi_{R}$. Since $\lambda_{1}\left(-\mathcal{L}_{0}, B_{R}\right)<0$, it is possible to normalize $\varphi_{R}$ in order to have:

$$
-\triangle\left(\eta \varphi_{R}\right) \leq f\left(\eta \varphi_{R}\right) \quad \text { in } B_{R}
$$

for all $\eta \in[0,1]$. Fix an arbitrary $x_{0} \in \mathbb{R}^{N}$ and denote, as usual, $\tau_{x_{0}} \varphi_{R}(\cdot):=\varphi_{R}\left(\cdot-x_{0}\right)$. Clearly $-\triangle\left(\tau_{x_{0}} \varphi_{R}\right) \leq f\left(\tau_{x_{0}} \varphi_{R}\right)$ in $B_{R}\left(x_{0}\right)$. Since $v>0$ in $\overline{B_{R}\left(x_{0}\right)}$, there exists $\underline{\eta} \in(0,1)$ such that $\eta \tau_{x_{0}} \varphi_{R}<v$ in $\overline{B_{R}\left(x_{0}\right)}$. Call $\eta^{*}=\sup \left\{\eta \in[0,1], \eta \tau_{x_{0}} \varphi_{R}<v\right.$ in $\left.\overline{B_{R}\left(x_{0}\right)}\right\}$. Assume $\eta^{*}<1$. Then $\eta^{*} \tau_{x_{0}} \varphi_{R} \leq v$ in $\overline{B_{R}\left(x_{0}\right)}$, with equality somewhere in $\overline{B_{R}\left(x_{0}\right)}$, and then in $B_{R}\left(x_{0}\right)$ because $\tau_{x_{0}} \varphi_{R}=0<v$ on $\partial B_{R}\left(x_{0}\right)$. But $-\triangle\left(\eta^{*} \varphi_{R}\right) \leq f\left(\eta^{*} \varphi_{R}\right)$ and $-\triangle v \geq f(v)$ 
in $B_{R}\left(x_{0}\right)$. The strong maximum principle then yields $\eta^{*} \tau_{x_{0}} \varphi_{R} \equiv v$ in $\overline{B_{R}\left(x_{0}\right)}$, which is impossible on the boundary. Thus, $\eta^{*}=1$ and $\tau_{x_{0}} \varphi_{R} \leq v$ in $\overline{B_{R}\left(x_{0}\right)}$. Hence,

$$
v\left(x_{0}\right) \geq \tau_{x_{0}} \varphi_{R}\left(x_{0}\right)=\varphi_{R}(0)>0
$$

and then $\inf _{\mathbb{R}^{N}} v \geq \varphi_{R}(0)$. Consequently $-\Delta v \geq f(v) \geq \inf _{\left[\varphi_{R}(0),+\infty\right)} f>0$. Applying Lemma 2.1 part (ii), with $\mathcal{L}=\triangle$ and $u \equiv 1$, we find a contradiction. This means that such a function $v$ cannot exist.

\subsection{The uniqueness result}

We now turn to the proof of Theorem 1.3 part 2). We follow the same ideas as in the proof of Theorem 1.1 part 2). We recall the sketch of that proof. First, we show that any positive bounded super-solution is bounded from below away from zero. Then, we establish a comparison principle between sub and a super-solutions (Theorem 3.6) by using the refined strong maximum principle. Note that here, we are not proving that any positive bounded super-solution $v$ satisfies $\inf v>0$, but we show that it is true for solutions. Therefore, under the assumptions of Theorem 1.3, we do not get the comparison principle for sub and super-solutions.

It is worth mentioning, without entering details, that under assumption (1.9) the refined strong maximum principle (Lemma 2.1) admits a much easier proof.

The following result represents the first step of the proof of Theorem 1.3 part 2).

Lemma 5.5 Let $u$ be a nonnegative bounded and not identically equal to 0 solution of (1.1). If (1.9) holds then either $\inf _{\mathbb{R}^{N}} u>0$, or there exists a limit operator with nonnegative principal eigenvalue in $\mathbb{R}^{N}$.

Proof. The strong maximum principle yields $u>0$ in $\mathbb{R}^{N}$. Suppose now that $\inf _{\mathbb{R}^{N}} u=0$. Hence there exists a sequence $\left(x_{n}\right)_{n \in \mathbb{N}}$ in $\mathbb{R}^{N}$ such that $\left|x_{n}\right| \rightarrow \infty$ and $u\left(x_{n}\right) \rightarrow 0$ as $n \rightarrow+\infty$. As in the proof of Proposition 5.3, set $u_{n}(x):=u\left(x_{n}+x\right) / u\left(x_{n}\right)$. For $x \in \mathbb{R}^{N}$ one has

$$
-a_{i j}\left(x_{n}+x\right) \partial_{i j} u_{n}(x)-q_{i}\left(x_{n}+x\right) \partial_{i} u_{n}(x)=g_{n}(x) u_{n}(x),
$$

where

$$
g_{n}(x):=\frac{f\left(x_{n}+x, u\left(x_{n}+x\right)\right)}{u\left(x_{n}+x\right)} .
$$

Note that the functions $g_{n}$ are uniformly bounded because $f$ is locally lipschitz-continuous in the second variable, uniformy in $x \in \mathbb{R}^{N}$. Since the $u_{n}$ satisfy (5.2) and $u_{n}(0)=1$, for any $R>0$ we can find, by the Harnack inequality, a constant $C_{R}>1$ such that

$$
\forall x \in B_{R}, \quad \forall n \in \mathbb{N}, \quad C_{R}^{-1} \leq u_{n}(x) \leq C_{R} .
$$

Thus, for $\xi \in B_{R}$, we have that

$$
0<u\left(x_{n}+\xi\right)=u_{n}(\xi) u\left(x_{n}\right) \leq C_{R} u\left(x_{n}\right)
$$


and then $\lim _{n \rightarrow \infty} u\left(x_{n}+\xi\right)=0$, uniformly in $\xi \in B_{R}$. Consequently

$$
\lim _{n \rightarrow \infty}\left(g_{n}(\xi)-f_{s}\left(x_{n}+\xi, 0\right)\right)=0
$$

uniformly in $\xi \in B_{R}$. Thanks to (1.9) we know that the functions $a_{i, j}\left(x_{n}+\cdot\right)$ and the $q_{i}\left(x_{n}+\cdot\right)$ are uniformly bounded in $C^{0, \gamma}\left(\mathbb{R}^{N}\right) \cap L^{\infty}\left(\mathbb{R}^{N}\right)$. Suppose temporarily that we know that the functions $g_{n}$ are uniformly bounded in $C^{0, \gamma}\left(B_{R}\right)$, for any $R>0$. Then, by standard interior estimates, it follows that, for any fixed $0<\tilde{\gamma}<\gamma$, the functions $u_{n}$ converge (up to subsequence) to some function $u^{*}$ in $C_{l o c}^{2, \tilde{\gamma}}\left(\mathbb{R}^{N}\right)$. Furthermore, by (1.9), we know that there exist $a_{i j}^{*}, q_{i}^{*}$ and $p^{*}$ such that

$$
a_{i j}\left(x_{n}+x\right) \rightarrow a_{i j}^{*}(x), \quad q_{i}\left(x_{n}+x\right) \rightarrow q_{i}^{*}(x), \quad f_{s}\left(x_{n}+x, 0\right) \rightarrow p^{*}(x)
$$

in $C_{\text {loc }}^{0, \tilde{\gamma}}\left(\mathbb{R}^{N}\right)$, as $n \rightarrow \infty$ (up to subsequence). Finally, letting (a subsequence of) $n$ go to infinity in (5.2) and using (5.4), one gets:

$$
\forall x \in \mathbb{R}^{N}, \quad-a_{i j}^{*}(x) \partial_{i j} u^{*}(x)-q_{i}^{*}(x) \partial_{i} u^{*}(x)=p^{*}(x) u^{*}(x) .
$$

By construction, $u^{*}$ is nonnegative and $u^{*}(0)=1$. Thus, the strong maximum principle implies that $u^{*}>0$ in $\mathbb{R}^{N}$. Recalling the definition (1.8), it follows that the principal eigenvalue in $\mathbb{R}^{N}$ of the limit operator $-a_{i j}^{*} \partial_{i j}-q_{i}^{*} \partial_{i}-p^{*}(x)$ is nonnegative.

To complete the proof, it only remains to show that the functions $g_{n}$ are uniformly bounded in $C^{0, \gamma}\left(B_{R}\right)$, for any $R>0$. Since the $u_{n}$ are uniformly bounded in $L^{\infty}\left(B_{R+1}\right)$, we can apply elliptic $L^{p}$-estimates and infer that the $u_{n}$ are uniformly bounded in $W^{2, p}\left(B_{R}\right)$, for any $p>1$. Thus, choosing $p$ big enough and using the imbedding theorem, we find out that the $u_{n}$ are uniformly bounded in $C^{0, \gamma}\left(B_{R}\right)$. By (5.3) we have that $u\left(x_{n}+x\right) \leq C_{R} u\left(x_{n}\right)$ for $x \in B_{R}$. There exists then $n_{0}$ such that $u\left(x_{n}+x\right) \leq \delta$ for all $x \in B_{R}$ and $n \geq n_{0}$, where $\delta>0$ is the positive constant (introduced in Section 1) such that $f(x, \cdot) \in C^{1}([0, \delta])$, uniformly in $x \in \mathbb{R}^{N}$. For $n \geq n_{0}$ and $x, y \in B_{R}$ with $x \neq y$, we have:

$$
\begin{aligned}
\frac{\left|g_{n}(x)-g_{n}(y)\right|}{|x-y|^{\gamma}} & \leq \frac{\left|f\left(x_{n}+x, u\left(x_{n}+x\right)\right)-f\left(x_{n}+y, u\left(x_{n}+x\right)\right)\right|}{u\left(x_{n}+x\right)|x-y|^{\gamma}} \\
& +\frac{\left|f\left(x_{n}+y, u\left(x_{n}+x\right)\right)\right|}{|x-y|^{\gamma}}\left|\frac{1}{u\left(x_{n}+x\right)}-\frac{1}{u\left(x_{n}+y\right)}\right| \\
& +\frac{\left|f\left(x_{n}+y, u\left(x_{n}+x\right)\right)-f\left(x_{n}+y, u\left(x_{n}+y\right)\right)\right|}{u\left(x_{n}+y\right)|x-y|^{\gamma}} .
\end{aligned}
$$

The first term of the right-hand side is controlled by the quantity

$$
\frac{1}{u\left(x_{n}+x\right)} \int_{0}^{u\left(x_{n}+x\right)} \frac{\left|f_{s}\left(x_{n}+x, t\right)-f_{s}\left(x_{n}+y, t\right)\right|}{|x-y|^{\gamma}} d t
$$

while the second and the third ones are controlled by

$$
k \frac{\left|u\left(x_{n}+x\right)-u\left(x_{n}+y\right)\right|}{\min \left(u\left(x_{n}+x\right), u\left(x_{n}+y\right)\right)|x-y|^{\gamma}},
$$


where $k$ is the lipschitz constant of the function $f\left(x_{n}+y, \cdot\right)$ in the set $\left[0,\|u\|_{\infty}\right]$ (which does not depend on $n$ and $y$ ). Consequently

$$
\frac{\left|g_{n}(x)-g_{n}(y)\right|}{|x-y|^{\gamma}} \leq \sup _{0 \leq s \leq \delta}\left\|f_{s}(\cdot, s)\right\|_{C^{0, \gamma\left(\mathbb{R}^{N}\right)}}+2 k \frac{\left|u\left(x_{n}+x\right)-u\left(x_{n}+y\right)\right|}{\min \left(u\left(x_{n}+x\right), u\left(x_{n}+y\right)\right)|x-y|^{\gamma}},
$$

and then we can conclude because, by (5.3),

$$
\frac{\left|u\left(x_{n}+x\right)-u\left(x_{n}+y\right)\right|}{\min \left(u\left(x_{n}+x\right), u\left(x_{n}+y\right)\right)|x-y|^{\gamma}}=\frac{\left|u_{n}(x)-u_{n}(y)\right|}{\min \left(u_{n}(x), u_{n}(y)\right)|x-y|^{\gamma}} \leq C_{R}\left\|u_{n}\right\|_{C^{0, \gamma}\left(B_{R}\right)} .
$$

Proof of Theorem 1.3 part 2). Let $u_{1}$ and $u_{2}$ be two bounded, nonnegative and not identically equal to 0 solutions of (1.1). Since the principal eigenvalue in $\mathbb{R}^{N}$ of every limit operator is negative we know, by Lemma 5.5, that inf $u_{1}>0$ and $\inf u_{2}>0$. Thus, we can apply Lemma 3.5 with $u=u_{1}$ and $v=u_{2}$ and next with $u=u_{2}$ and $v=u_{1}$. We conclude that $u_{1} \equiv u_{2}$.

\subsection{Proof of Theorem 1.5: the almost periodic case}

We prove Theorem 1.5 showing preliminarily that, in the a.p. framework, $\lambda_{1}\left(-\mathcal{L}^{*}, \mathbb{R}^{N}\right)=$ $\lambda_{1}\left(-\mathcal{L}_{0}, \mathbb{R}^{N}\right)$ for any limit operator $-\mathcal{L}^{*}$. This is done in the next lemma. Then, we conclude applying Theorem 1.3.

Lemma 5.6 Assume that (1.9) holds and that the functions $a_{i j}, q_{i}$ and $f_{s}(x, 0)$ are a.p. Then $\lambda_{1}\left(-\mathcal{L}^{*}, \mathbb{R}^{N}\right)=\lambda_{1}\left(-\mathcal{L}_{0}, \mathbb{R}^{N}\right)$ for any limit operator $-\mathcal{L}^{*}$.

Proof. Let $-\mathcal{L}^{*}=-a_{i j}^{*} \partial_{i j}-q_{i}^{*} \partial_{i}-p^{*}$ be a limit operator (associated with $-\mathcal{L}_{0}$ ). Thanks to Proposition 5.3, we have that $\lambda_{1}\left(-\mathcal{L}^{*}, \mathbb{R}^{N}\right) \geq \lambda_{1}\left(-\mathcal{L}_{0}, \mathbb{R}^{N}\right)$.

We prove the opposite inequality showing that $-\mathcal{L}_{0}$ is a limit operator associated with $-\mathcal{L}^{*}$, and then applying Proposition 5.3. By definition, there exists a sequence $\left(x_{n}\right)_{n \in \mathbb{N}}$ in $\mathbb{R}^{N}$ such that: $\left|x_{n}\right| \rightarrow \infty$ and, for $x \in \mathbb{R}^{N}$,

$$
a_{i j}\left(x_{n}+x\right) \rightarrow a_{i j}^{*}(x), \quad q_{i}\left(x_{n}+x\right) \rightarrow q_{i}^{*}(x), \quad f_{s}\left(x_{n}+x, 0\right) \rightarrow p^{*}(x) .
$$

Since the functions $a_{i j}, q_{i}$ and $f_{s}(\cdot, 0)$ are a.p, we have that the above convergences hold (up to subsequence) uniformly in $x \in \mathbb{R}^{N}$. Furthermore, it is easy to check that

$\left\|a_{i j}^{*}\right\|_{C^{0, \gamma}\left(\mathbb{R}^{N}\right)} \leq\left\|a_{i j}\right\|_{C^{0, \gamma\left(\mathbb{R}^{N}\right)}}, \quad\left\|q_{i}^{*}\right\|_{C^{0, \gamma}\left(\mathbb{R}^{N}\right)} \leq\left\|q_{i}\right\|_{C^{0, \gamma}\left(\mathbb{R}^{N}\right)}, \quad\left\|p^{*}\right\|_{C^{0, \gamma}\left(\mathbb{R}^{N}\right)} \leq\left\|f_{s}(\cdot, 0)\right\|_{C^{0, \gamma}\left(\mathbb{R}^{N}\right)}$.

Thus, up to subsequence, the functions $a_{i j}^{*}\left(-x_{n}+x\right), q_{i}^{*}\left(-x_{n}+x\right)$ and $p^{*}\left(-x_{n}+x\right)$ converge in $C_{\text {loc }}^{0, \tilde{\gamma}}\left(\mathbb{R}^{N}\right)$ as $n$ goes to infinity, for some $0<\tilde{\gamma}<\gamma$. We have that $\left|-x_{n}\right| \rightarrow+\infty$ and, by the uniform convergence of $a_{i j}\left(x_{n}+x\right)$ to $a_{i j}^{*}(x)$, that:

$$
\forall x \in \mathbb{R}^{N}, \quad a_{i j}(x)=\lim _{n \rightarrow \infty} a_{i j}\left(x_{n}-x_{n}+x\right)=\lim _{n \rightarrow \infty} a_{i j}^{*}\left(-x_{n}+x\right) .
$$


Analogously,

$$
\forall x \in \mathbb{R}^{N}, \quad q_{i}(x)=\lim _{n \rightarrow \infty} q_{i}^{*}\left(-x_{n}+x\right), \quad f_{s}(x, 0)=\lim _{n \rightarrow \infty} p^{*}\left(-x_{n}+x\right)
$$

and then $-\mathcal{L}_{0}$ is a limit operator associated with $-\mathcal{L}^{*}$. Applying Proposition 5.3 to the elliptic operator $-\mathcal{L}^{*}$ we find: $\lambda_{1}\left(-\mathcal{L}_{0}, \mathbb{R}^{N}\right) \geq \lambda_{1}\left(-\mathcal{L}^{*}, \mathbb{R}^{N}\right)$.

Theorem 1.5 follows immediately from Lemma 5.6 and Theorem 1.3 part 2).

We conclude this section with a sufficient condition on $f(x, s)$ which guarantees the almost periodicity of $f_{s}(x, 0)$.

Proposition 5.7 If for any $s \in[0, \delta]$ the function $x \mapsto f(x, s)$ is a.p, then $f_{s}(x, 0)$ is a.p. too.

Proof. Consider an arbitrary sequence $\left(x_{n}\right)_{n \in \mathbb{N}}$ in $\mathbb{R}^{N}$. By the a.p. assumption on $f$, for any $m \in \mathbb{N}$ such that $1 / m<\delta$, we can find a subsequence $\left(x_{n}^{m}\right)_{n \in \mathbb{N}}$ of $\left(x_{n}\right)_{n \in \mathbb{N}}$ such that $f\left(x_{n}^{m}+x, 1 / m\right)$ converges uniformly in $x \in \mathbb{R}^{N}$ as $n$ goes to infinity. Thus, using a diagonal method, we find a common subsequence $\left(x_{n_{k}}\right)_{k \in \mathbb{N}}$ such that, for any $m \in \mathbb{N}$ satisfying $1 / m<\delta, f\left(x_{n_{k}}+x, 1 / m\right)$ converges uniformly in $x \in \mathbb{R}^{N}$ as $k$ goes to infinity. We want to prove that $f_{s}\left(x_{n_{k}}+x, 0\right)$ converges uniformly in $x \in \mathbb{R}^{N}$. Since $s \mapsto f(x, s)$ is in $C^{1}([0, \delta])$ uniformly in $x \in \mathbb{R}^{N}$, for any fixed $\varepsilon>0$ there exists $m \in \mathbb{N}$ such that:

$$
\frac{1}{m}<\delta, \quad \sup _{x \in \mathbb{R}^{N}}\left|f_{s}(x, 0)-m f\left(x, \frac{1}{m}\right)\right|<\frac{\varepsilon}{3} .
$$

Therefore, for $h, l \in \mathbb{N}, h, l$ big enough, we have:

$\sup _{x \in \mathbb{R}^{N}}\left|f_{s}\left(x_{n_{h}}+x, 0\right)-f_{s}\left(x_{n_{l}}+x, 0\right)\right|<\frac{2}{3} \varepsilon+m \sup _{x \in \mathbb{R}^{N}}\left|f\left(x_{n_{h}}+x, \frac{1}{m}\right)-f\left(x_{n_{l}}+x, \frac{1}{m}\right)\right|<\varepsilon$,

where the last inequality follows from the uniform convergence of $f\left(x_{n_{k}}+x, 1 / m\right)$.

\section{$6 \quad$ Necessary conditions}

\subsection{A generalization of the notion of periodic principal eigen- value: $\lambda_{1}^{\prime}$}

The necessary conditions presented in this section involve the quantity

$$
\lambda_{1}^{\prime}\left(-L, \mathbb{R}^{N}\right):=\inf \left\{\lambda \mid \exists \phi \in C^{2}\left(\mathbb{R}^{N}\right) \cap W^{1, \infty}\left(\mathbb{R}^{N}\right), \phi>0,-(L+\lambda) \phi \leq 0 \text { in } \mathbb{R}^{N}\right\},
$$

where $-L$ stands, as usual, for a generic elliptic operator with coefficients in $C_{\text {loc }}^{0, \gamma}\left(\mathbb{R}^{N}\right) \cap$ $L^{\infty}\left(\mathbb{R}^{N}\right)$. From the definition (6.1) of $\lambda_{1}^{\prime}$ it follows immediately the necessary condition.

Proposition 6.1 Let $\lambda_{1}^{\prime}\left(-\mathcal{L}_{0}, \mathbb{R}^{N}\right)$ be the quantity defined by (6.1) and assume that

$$
\forall x \in \mathbb{R}^{N}, \forall s \geq 0, \quad f(x, s) \leq f_{s}(x, 0) s .
$$

If $\lambda_{1}^{\prime}\left(-\mathcal{L}_{0}, \mathbb{R}^{N}\right)>0$, then there is no nonnegative bounded solution of (1.1), other than the trivial one $u \equiv 0$. 
Proof. Let $u$ be a nonnegative bounded solution of $(1.1)$. Since $f(x, \cdot)$ is locally lipschitzcontinuous, uniformly in $x \in \mathbb{R}^{N}$, we can apply the strong maximum principle and infer that either $u \equiv 0$, or $u>0$ in $\mathbb{R}^{N}$. Suppose by a contradiction that $u>0$. By (6.2) we have that

$$
\forall x \in \mathbb{R}^{N}, \quad-a_{i j}(x) \partial_{i j} u-q_{i}(x) \partial_{i} u=f(x, u) \leq f_{s}(x, 0) u .
$$

Remembering the definition (6.1), we conclude that $\lambda_{1}^{\prime}\left(-\mathcal{L}_{0}, \mathbb{R}^{N}\right) \leq 0$.

Remark 6.2 If we replace $\lambda_{1}^{\prime}\left(-\mathcal{L}_{0}, \mathbb{R}^{N}\right)$ with the quantity

$$
\lambda_{1}^{\prime \prime}\left(-\mathcal{L}_{0}, \mathbb{R}^{N}\right):=\sup \left\{\lambda \mid \exists \phi \in C^{2}\left(\mathbb{R}^{N}\right) \cap W^{1, \infty}\left(\mathbb{R}^{N}\right), \phi>0,\left(\mathcal{L}_{0}+\lambda\right) \phi \leq 0 \text { in } \mathbb{R}^{N}\right\},
$$

(which is more natural, in that it is more similar to $\lambda_{1}\left(-\mathcal{L}_{0}, \mathbb{R}^{N}\right)$ ) the necessary condition given by Proposition 6.1 does not hold anymore. Consider, in fact, the equation - $u^{\prime \prime}-$ $q(x) u^{\prime}=0$ in $\mathbb{R}$. We show that, if $q$ is opportunely chosen, there exists a positive function $\phi \in C^{2}(\mathbb{R}) \cap W^{1, \infty}(\mathbb{R})$ such that $\left(\mathcal{L}_{0}+1\right) \phi \leq 0$ in $\mathbb{R}\left(\mathcal{L}_{0} u=u^{\prime \prime}+q(x) u^{\prime}\right.$ in this case). Therefore $\lambda_{1}^{\prime \prime}\left(-\mathcal{L}_{0}, \mathbb{R}\right) \geq 1$, but all the functions $u$ identically equal to a positive constant solve $-u^{\prime \prime}-q(x) u^{\prime}=0$. The function $\phi$ is defined in $[-1,1]$ by: $\phi(x)=2-x^{2}$. For $x \in(-1,1)$ we have that $\left(\mathcal{L}_{0}+1\right) \phi=-2-2 q(x) x+\phi \leq-2 q(x) x$, so it is sufficient to take $q(x) \leq 0$ for $x \leq 0$ and $q(x) \geq 0$ for $x \geq 0$ to have: $\left(\mathcal{L}_{0}+1\right) \phi \leq 0$ in $(-1,1)$. Then set

$$
\phi(x):= \begin{cases}e^{x} & \text { if } x \leq-2 \\ e^{-x} & \text { if } x \geq 2\end{cases}
$$

For $x<-2,\left(\mathcal{L}_{0}+1\right) \phi=e^{x}(2+q(x))$ and, for $x>2,\left(\mathcal{L}_{0}+1\right) \phi=e^{-x}(2-q(x))$. Hence, if $q \leq-2$ for $x<-2$ and $q(x)>2$ for $x>2$, we find: $\left(\mathcal{L}_{0}+1\right) \phi \leq 0$ in $(-\infty,-2) \cup(2,+\infty)$. Clearly, it is possible to define $\phi$ in $(-2,-1) \cup(1,2)$ in such a way that $\inf _{[-2,-1]} \phi^{\prime}>0$, $\sup _{[1,2]} \phi^{\prime}<0$ and $\phi \in C^{2}(\mathbb{R}) \cap W^{1, \infty}(\mathbb{R})$. Consequently, taking $q$ sufficiently negative in $(-2,-1)$ and sufficiently positive in $(1,2)$, we get: $\left(\mathcal{L}_{0}+1\right) \phi \leq 0$ in $\mathbb{R}$.

We prove now that if $L$ is periodic in $x$, in the sense that its coefficients are periodic in $x$ with the same period $\left(l_{1}, \ldots, l_{N}\right)$, then $\lambda_{1}^{\prime}\left(-L, \mathbb{R}^{N}\right)$ coincides with the periodic principal eigenvalue $\lambda_{p}$ of $-L$. The periodic principal eigenvalue is defined as the unique real number $\lambda_{p}$ such that there exists a periodic function $\varphi_{p} \in C^{2}\left(\mathbb{R}^{N}\right)$ (with the same period as the coefficients of $L$ ) satisfying:

$$
\left\{\begin{array}{cl}
\left(L+\lambda_{p}\right) \varphi_{p}=0 & \text { in } \mathbb{R}^{N} \\
\varphi_{p}>0 & \text { in } \mathbb{R}^{N}
\end{array}\right.
$$

The function $\varphi_{p}$, which is called periodic principal eigenfunction, is unique up to multiplication. The proof of the fact that $\lambda_{1}^{\prime}\left(-L, \mathbb{R}^{N}\right)=\lambda_{p}$ is a direct application of Lemma 2.1 .

Proposition 6.3 If $L$ is periodic, then the periodic principal eigenvalue $\lambda_{p}$ given by (6.4) coincides with $\lambda_{1}^{\prime}\left(-L, \mathbb{R}^{N}\right)$. 
Proof. Taking $\phi=\varphi_{p}$ in (6.1), we obtain that $\lambda_{1}^{\prime}\left(-L, \mathbb{R}^{N}\right) \leq \lambda_{p}$. Consider an arbitrary $\lambda<\lambda_{p}$. Since $\inf _{\mathbb{R}^{N}} \varphi_{p}>0$, one can find a positive constant $\varepsilon$ such that

$$
-(L+\lambda) \varphi_{p}=\left(\lambda_{p}-\lambda\right) \varphi_{p} \geq \varepsilon .
$$

Applying statement (ii) of Lemma 2.1 to the operator $\mathcal{L}=L+\lambda$ (with $v=\varphi_{p}$ ), we infer that any function $\phi \in C^{2}\left(\mathbb{R}^{N}\right) \cap L^{\infty}\left(\mathbb{R}^{N}\right)$, which satisfies $-(L+\lambda) \phi \leq 0$, is nonpositive. Since this is true for any $\lambda<\lambda_{p}$, we conclude that $\lambda_{1}^{\prime}\left(-L, \mathbb{R}^{N}\right) \geq \lambda_{p}$.

The result of Proposition 6.3 can be extended to general periodic domains. It is known that, if $\Omega$ is a smooth and periodic domain, with the same period $\left(l_{1}, \cdots, l_{N}\right)$ as $L^{2}$, then there exist two numbers $\lambda_{p, D}, \lambda_{p, N}$ and two $C^{2}(\bar{\Omega})$ functions $\varphi_{p, D}, \varphi_{p, N}$ such that

$$
\begin{gathered}
\left\{\begin{array}{cl}
\left(L+\lambda_{p, D}\right) \varphi_{p, D}=0 & \text { in } \Omega, \\
\varphi_{p, D}>0 & \text { in } \Omega, \\
\varphi_{p, D}=0 & \text { on } \partial \Omega, \\
\varphi_{p, D} & \text { is periodic, }
\end{array}\right. \\
\left\{\begin{array}{cl}
\left(L+\lambda_{p, N}\right) \varphi_{p, N}=0 & \text { in } \Omega, \\
\varphi_{p, N}>0 & \text { in } \bar{\Omega}, \\
\partial_{\nu} \varphi_{p, N}=0 & \text { on } \partial \Omega, \\
\varphi_{p, N} & \text { is periodic. }
\end{array}\right.
\end{gathered}
$$

The real numbers $\lambda_{p, D}$ and $\lambda_{p, N}$ are unique and they are called periodic principal eigenvalues of $-L$ in $\Omega$, respectively under Dirichlet and under Neumann boundary conditions. The functions $\varphi_{p, D}$ and $\varphi_{p, N}$ are unique up to multiplication. Define the following quantities:

$$
\begin{aligned}
& \lambda_{1, D}^{\prime}(-L, \Omega):=\inf \left\{\lambda \mid \exists \phi \in E_{D},-(L+\lambda) \phi \leq 0 \text { in } \Omega\right\}, \\
& \lambda_{1, N}^{\prime}(-L, \Omega):=\inf \left\{\lambda \mid \exists \phi \in E_{N},-(L+\lambda) \phi \leq 0 \text { in } \Omega\right\},
\end{aligned}
$$

where

$$
\begin{gathered}
E_{D}=\left\{\phi \in C^{2, b}(\bar{\Omega}) \text { for some } 0<b<1, \phi>0 \text { in } \Omega, \phi=0 \text { on } \partial \Omega\right\}, \\
E_{N}=\left\{\phi \in C^{2, b}(\bar{\Omega}) \text { for some } 0<b<1, \phi>0 \text { in } \Omega, \partial_{\nu} \phi \leq 0 \text { on } \partial \Omega\right\}
\end{gathered}
$$

and $\nu$ denotes the outward unit normal on $\partial \Omega$. By $C^{2, b}(\bar{\Omega})$, we mean the set of $C^{2}(\bar{\Omega})$ functions $\phi$ with bounded derivatives up to the second order, and for which $\partial_{i j} \phi \in C^{0, b}(\bar{\Omega})$ for all $1 \leq i, j \leq N$.

Proposition 6.4 Assume that $\Omega$ is a smooth domain satisfying, for $i=1, \ldots, N, \Omega+$ $\left\{l_{i} e_{i}\right\}=\Omega$ and assume that the coefficients of $L$ are in $C^{0, \gamma}(\bar{\Omega})$ and are periodic in $x$, with period $\left(l_{1}, \ldots, l_{N}\right)$. Then $\lambda_{1, D}^{\prime}(-L, \Omega)=\lambda_{p, D}$ and $\lambda_{1, N}^{\prime}(-L, \Omega)=\lambda_{p, N}$.

Proof. Since $\varphi_{p, D} \in E_{D}$ and $\varphi_{p, N} \in E_{N}$ (in fact $\varphi_{p, D}$ and $\varphi_{p, N}$ are periodic and of class $C^{2, \gamma}(\bar{\Omega})$ for some $\gamma>0$ by elliptic estimates up to the boundary) we get immediately: $\lambda_{1, D}^{\prime}(-L, \Omega) \leq \lambda_{p, D}$ and $\lambda_{1, N}^{\prime}(-L, \Omega) \leq \lambda_{p, N}$.

\footnotetext{
${ }^{2}$ in the sense that, for $i \in\{1, \cdots, N\}, \Omega+\left\{l_{i} e_{i}\right\}=\Omega$
} 
Suppose by a contradiction that $\lambda_{1, D}^{\prime}(-L, \Omega)<\lambda_{p, D}$. There exist then $\phi \in E_{D}$ and $\lambda<\lambda_{p, D}$ such that $-(L+\lambda) \phi \leq 0$ in $\Omega$. We show now that it is possible to find a positive number $k$ such that $\varphi_{p, D} \geq k \phi$ in $\bar{\Omega}$. If not, in fact, there exists a sequence $\left(x_{n}\right)_{n \in \mathbb{N}}$ in $\bar{\Omega}$ verifying:

$$
\varphi_{p, D}\left(x_{n}\right)<\phi\left(x_{n}\right) / n \text {. }
$$

Note that $x_{n} \in \Omega$ because $\phi \leq 0=\varphi_{p, D}$ on $\partial \Omega$. Since $\varphi_{p, D}$ is periodic, for every $n \in \mathbb{N}$ there exists $y_{n} \in \Omega \cap\left[0, l_{1}\right] \times \ldots \times\left[0, l_{N}\right]$ such that $\varphi_{p, D}\left(y_{n}\right)=\varphi_{p, D}\left(x_{n}\right)$. Consider the limit $y$ of a converging subsequence of $\left(y_{n}\right)_{n \in \mathbb{N}}$ (that we still call $\left(y_{n}\right)$ ) and, for $n \in \mathbb{N}$, consider a point $z_{n} \in \partial \Omega$ such that $t_{n}:=\operatorname{dist}\left(y_{n}, \partial \Omega\right)=\left|y_{n}-z_{n}\right|>0$. We have that

$$
\varphi_{p, D}(y)=\lim _{n \rightarrow \infty} \varphi_{p, D}\left(x_{n}\right) \leq \lim _{n \rightarrow \infty} \frac{1}{n} \phi\left(x_{n}\right)=0,
$$

which yields: $y \in \partial \Omega$ and $\lim _{n \rightarrow \infty} t_{n}=0$. It is easy to check that $y_{n}=z_{n}-t_{n} \nu\left(z_{n}\right)$ for any $n \in \mathbb{N}$. Hence there exists $0<s_{n}<t_{n}$ such that

$$
-\nabla \varphi_{p, D}\left(z_{n}-s_{n} \nu\left(z_{n}\right)\right) \cdot \nu\left(z_{n}\right)=\frac{\varphi_{p, D}\left(y_{n}\right)}{t_{n}}<\frac{1}{n} \frac{\phi\left(x_{n}\right)}{t_{n}} \leq \frac{1}{n} \sqrt{\sum_{i=1}^{N}\left\|\partial_{i} \phi\right\|_{L^{\infty}(\Omega)}^{2}}
$$

where the last inequality holds because $t_{n}=\operatorname{dist}\left(x_{n}, \partial \Omega\right)>0$ and $\phi \leq 0$ on $\partial \Omega$. Letting $n$ go to infinity in $(6.5)$ we get that $-\partial_{\nu} \varphi_{p, D}(y) \leq 0$. That is a contradiction with the Hopf's lemma.

Therefore, there exists $k>0$ such that $\varphi_{p, D} \geq k \phi$ in $\bar{\Omega}$. Consider now the number

$$
k^{*}:=\sup \left\{k>0 \mid \varphi_{p, D}-k \phi \geq 0 \text { in } \bar{\Omega}\right\} .
$$

Clearly, $k^{*}<\infty$ and the function $\psi:=\varphi_{p, D}-k^{*} \phi$ satisfies:

$$
\psi \geq 0, \quad-(L+\lambda) \psi \geq 0 \quad \text { in } \Omega,
$$

and there exists a sequence $\left(\xi_{n}\right)_{n \in \mathbb{N}}$ in $\bar{\Omega}$ such that $\psi\left(\xi_{n}\right)<\phi\left(\xi_{n}\right) / n$. For $n \in \mathbb{N}$ consider $\eta_{n} \in l_{1} \mathbb{Z} \times \cdots \times l_{N} \mathbb{Z}$ such that

$$
c_{n}:=\xi_{n}-\eta_{n} \in \bar{\Omega} \cap\left[0, l_{1}\right] \times \ldots \times\left[0, l_{N}\right]
$$

and define $\psi_{n}(x):=\psi\left(x+\eta_{n}\right)$ for all $x \in \bar{\Omega}$. Consequently, $\psi_{n}\left(c_{n}\right)<\phi\left(\xi_{n}\right) / n$ and the sequence $\left(\psi_{n}\right)_{n \in \mathbb{N}}$ converges, as $n \rightarrow+\infty$ and up to subsequence, to a function $\tilde{\psi}$ in $C^{2, b}\left(\overline{\Omega \cap B_{R}}\right)$ (for some $b>0)$, where $R$ is chosen such that $\left[0, l_{1}\right] \times \ldots \times\left[0, l_{N}\right] \subset B_{R}$. Moreover, using the periodicity of $L$, we find that $-(L+\lambda) \tilde{\psi} \geq 0$ in $\Omega \cap B_{R}$. Let $c \in \bar{\Omega} \cap\left[0, l_{1}\right] \times \ldots \times\left[0, l_{N}\right]$ be the limit of a converging subsequence of $\left(c_{n}\right)$. We have that $\tilde{\psi}(c)=0$.

If $\tilde{\psi}>0$ in $\Omega \cap B_{R}$ then $c \in \partial \Omega$. Proceeding as above we can then find a sequence $\left(\theta_{n}\right)_{n \in \mathbb{N}}$ of points in $\partial \Omega$ and two sequences of positive numbers $\left(s_{n}\right)_{n \in \mathbb{N}}$ and $\left(t_{n}\right)_{n \in \mathbb{N}}$ such that: $\theta_{n} \rightarrow c$, $s_{n}, t_{n} \rightarrow 0$ as $n \rightarrow+\infty$, and

$$
-\nabla \psi_{n}\left(\theta_{n}-s_{n} \nu\left(\theta_{n}\right)\right) \cdot \nu\left(\theta_{n}\right)=\frac{\psi_{n}\left(c_{n}\right)}{t_{n}}<\frac{1}{n} \frac{\phi\left(\xi_{n}\right)}{t_{n}} \leq \frac{1}{n} \sqrt{\sum_{i=1}^{N}\left\|\partial_{i} \phi\right\|_{L^{\infty}(\Omega)}^{2}} .
$$


Passing to the limit as $n$ goes to infinity we obtain that $-\partial_{\nu} \tilde{\psi}(c) \leq 0$, which contradicts the Hopf's lemma.

If there exists $x_{0} \in \Omega \cap B_{R}$ such that $\tilde{\psi}\left(x_{0}\right)=0$, then we can find $\rho>0$ such that $B_{\rho}\left(x_{0}\right) \subset \Omega \cap B_{R}$ and $\tilde{\psi}=0$ in $B_{\rho}\left(x_{0}\right)$. This means that $k^{*} \phi\left(\cdot+\eta_{n}\right)$ converges to $\varphi_{p, D}$ in $C^{2, b}\left(\overline{B_{\rho}\left(x_{0}\right)}\right)$. Thus, we find the following contradiction:

$$
-L \varphi_{p, D}\left(x_{0}\right)=k^{*} \lim _{n \rightarrow \infty}\left(-L \phi\left(x_{0}+\eta_{n}\right)\right) \leq k^{*} \lim _{n \rightarrow \infty} \lambda \phi\left(x_{0}+\eta_{n}\right)<\lambda_{p, D} \varphi_{p, D}\left(x_{0}\right) .
$$

The proof for the case with Neumann boundary conditions is similar. We suppose, by a contradiction, that $\lambda_{1, N}^{\prime}(-L, \Omega)<\lambda_{p, N}$ and we take $\phi \in E_{N}$ and $\lambda<\lambda_{p, N}$ such that $-(L+\lambda) \phi \leq 0$ in $\Omega$. We set

$$
k^{*}:=\sup \left\{k>0 \mid \varphi_{p, N}-k \phi \geq 0 \text { in } \bar{\Omega}\right\}
$$

and we proceed as in the previous case, with $\varphi_{p, D}$ replaced by $\varphi_{p, N}$. The only difference is when $\tilde{\psi}>0$ in $\Omega \cap B_{R}$ and $c \in \partial \Omega$. In this case we obtain:

$$
\partial_{\nu} \tilde{\psi}(c)=\lim _{n \rightarrow+\infty} \partial_{\nu} \psi_{n}(c)=\partial_{\nu} \varphi_{p, N}(c)-k^{*} \lim _{n \rightarrow+\infty} \partial_{\nu} \phi\left(c+\eta_{n}\right) \geq 0,
$$

which is the usual contradiction with the Hopf's lemma.

As shown by next result, the condition $\lambda_{1}\left(-\mathcal{L}_{0}, \mathbb{R}^{N}\right)<0$ in Theorem 5.1 can be replaced by $\lambda_{1}^{\prime}\left(-\mathcal{L}_{0}, \mathbb{R}^{N}\right)<0$. On the other hand, we know that, under assumption (6.2), the positivity of $\lambda_{1}^{\prime}\left(-\mathcal{L}_{0}, \mathbb{R}^{N}\right)$ implies the nonexistence of positive bounded solutions of (1.1). As already mentioned in the introduction, we do not know in general what happens when $\lambda_{1}^{\prime}\left(-\mathcal{L}_{0}, \mathbb{R}^{N}\right)=0$.

Theorem 6.5 If (1.4) holds and $\lambda_{1}^{\prime}\left(-\mathcal{L}_{0}, \mathbb{R}^{N}\right)<0$, then there exists at least one positive bounded solution of (1.1).

Proof. If $\lambda_{1}^{\prime}\left(-\mathcal{L}_{0}, \mathbb{R}^{N}\right)<0$, there exists a constant $\lambda<0$ and a positive bounded function $\phi$ such that $-\mathcal{L}_{0} \phi \leq \lambda \phi$ in $\mathbb{R}^{N}$. Hence, the function $\phi$, opportunely normalized, is a subsolution of (1.1) and we can then proceed as in the proof of Theorem 5.1.

\subsection{Comparison between $\lambda_{1}$ and $\lambda_{1}^{\prime}$}

In the last part of this section we establish some comparisons between the quantities $\lambda_{1}\left(-L, \mathbb{R}^{N}\right)$ and $\lambda_{1}^{\prime}\left(-L, \mathbb{R}^{N}\right)$ (= $\lambda_{p}$ in the periodic case) in different situations, focalizing in particular on the self adjoint case. We say that the elliptic operator $-L$ is self adjoint if it is of the form

$$
-L u=-\partial_{i}\left(a_{i j}(x) \partial_{j} u\right)-c(x) u,
$$

with $\left(a_{i j}\right)_{i, j}$ symmetric. In that case we assume that the $a_{i j}$ are in $C_{l o c}^{1, \gamma}\left(\mathbb{R}^{N}\right)$.

We start with a result concerning self adjoint periodic operators which follows from Propositions 4.2, 6.3 and from a standard result. This standard result states that the sequence of principal eigenvalues of $-L$ in $B_{R}$, with Dirichlet boundary conditions, converges to the periodic principal eigenvalue $\lambda_{p}$ as $R$ goes to infinity (see e.g. Lemma 3.6 in [4]). We include its proof here for the sake of completeness. 
Proposition 6.6 If the operator $-L$ is self adjoint and periodic, then $\lambda_{1}\left(-L, \mathbb{R}^{N}\right)=$ $\lambda_{1}^{\prime}\left(-L, \mathbb{R}^{N}\right)=\lambda_{p}$, where $\lambda_{p}$ is the periodic principal eigenvalue given by (6.4).

Proof. First, one knows from Proposition 6.3 that $\lambda_{p}=\lambda_{1}^{\prime}\left(-L, \mathbb{R}^{N}\right)$. Let now $\varphi_{p}$ be the periodic principal eigenfunction of $-L$ in $\mathbb{R}^{N}$. Taking $\phi=\varphi_{p}$ in (1.8), it follows immediately that $\lambda_{1}\left(-L, \mathbb{R}^{N}\right) \geq \lambda_{p}$.

To show the opposite inequality consider a family of functions $\left(\chi_{R}\right)_{R>2}$ uniformly bounded in $C^{2}\left(\mathbb{R}^{N}\right)$, such that $\chi_{R} \in C_{0}^{2}\left(B_{R}\right)$ and $\chi_{R}=1$ in $B_{R-1}$. The set $C_{0}^{2}\left(B_{R}\right)$ denotes the set of $C^{2}\left(\overline{B_{R}}\right)$ functions vanishing on $\partial B_{R}$.

Fix $R>0$ and call $\lambda_{R}$ the principal eigenvalue of $-L$ in $B_{R}$. As known, the following variational formula holds:

$$
\lambda_{R}=\min \frac{\int_{B_{R}}\left(\partial_{i} v a_{i j}(x) \partial_{j} v-c(x) v^{2}\right)}{\int_{B_{R}} v^{2}},
$$

where the minimum is taken over all nonzero functions $v \in C_{0}^{2}\left(B_{R}\right)$. Taking $v=\varphi_{p} \chi_{R}$ as a test function in (6.6), and calling $C_{R}=B_{R} \backslash B_{R-1}$, we find:

$$
\begin{gathered}
\lambda_{R} \leq-\frac{\int_{B_{R}}\left(L\left(\varphi_{p} \chi_{R}\right)\right) \varphi_{p} \chi_{R}}{\int_{B_{R}} \varphi_{p}^{2} \chi_{R}^{2}}=\frac{\lambda_{p} \int_{B_{R-1}} \varphi_{p}^{2}-\int_{C_{R}}\left(L\left(\varphi_{p} \chi_{R}\right)\right) \varphi_{p} \chi_{R}}{\int_{B_{R}} \varphi_{p}^{2} \chi_{R}^{2}} \\
=\lambda_{p}-\frac{\lambda_{p} \int_{C_{R}} \varphi_{p}^{2} \chi_{R}^{2}+\int_{C_{R}}\left(L\left(\varphi_{p} \chi_{R}\right)\right) \varphi_{p} \chi_{R}}{\int_{B_{R}} \varphi_{p}^{2} \chi_{R}^{2}} .
\end{gathered}
$$

Since $\min \varphi_{p}>0$, it follows that there exists $K>0$ such that

$$
\int_{B_{R}} \varphi_{p}^{2} \chi_{R}^{2} \geq \int_{B_{R-1}} \varphi_{p}^{2} \geq K(R-1)^{N},
$$

for $R>1$. Consequently,

$$
\lambda_{R} \leq \lambda_{p}+K^{\prime} \frac{R^{N-1}}{(R-1)^{N}},
$$

where $K^{\prime}$ is a positive constant independent on $R$. Letting $R$ go to infinity and using Proposition 4.2, we get: $\lambda_{1}\left(-L, \mathbb{R}^{N}\right) \leq \lambda_{p}$ and then $\lambda_{1}\left(-L, \mathbb{R}^{N}\right)=\lambda_{p}$.

Proposition 6.6, together with Proposition 6.3, allows to prove that Theorem 1.3 is a generalization of the results already known in the periodic self adjoint case. In fact:

Proposition 6.7 Let $L$ be a periodic operator with continuous coefficients and let $-L^{*}$ be a limit operator associated with $-L$. Then $\lambda_{p}=\lambda_{p}^{*}$, where $\lambda_{p}$ and $\lambda_{p}^{*}$ are the periodic principal eigenvalue in $\mathbb{R}^{N}$ respectively of $-L$ and $-L^{*}$. Furthermore, if $L$ is self adjoint, then $\lambda_{1}\left(-L^{*}, \mathbb{R}^{N}\right)=\lambda_{p}=\lambda_{1}\left(-L, \mathbb{R}^{N}\right)$. 
Proof. The constant $\lambda_{p}^{*}$ is well defined because $-L^{*}$ has the same periodicity as $-L$. Let $\left(x_{n}\right)_{n \in \mathbb{N}}$ be the sequence given by the Definition 1.2, such that $\lim _{n \rightarrow \infty} L\left(x_{n}+x\right)=L^{*}(x)$ for $x \in \mathbb{R}^{N}$. By the periodicity of $L$, we can find a sequence $\left(y_{n}\right)_{n \in \mathbb{N}}$ in $\left[0, l_{1}\right] \times \cdots \times\left[0, l_{N}\right]$ such that $L^{*}(\cdot)=\lim _{n \rightarrow \infty} L\left(y_{n}+\cdot\right)$. Let $y$ be the limit of (a subsequence of) $\left(y_{n}\right)_{n \in \mathbb{N}}$. Hence $L^{*}(\cdot)=L(y+\cdot)$. Therefore the periodic principal eigenfunction of $-L^{*}$ is equal to the periodic principal eigenfunction of $-L$ translated by $y$, with the same periodic principal eigenvalue. The last assertion follows immediately from Proposition 6.6 because, if $L$ is self adjoint, so is $L^{*}$.

Proposition 6.6 shows that $\lambda_{1}\left(-L, \mathbb{R}^{N}\right)=\lambda_{p}$ in the periodic self adjoint case. In the periodic non-self adjoint case we always have

$$
\lambda_{1}\left(-L, \mathbb{R}^{N}\right) \geq \lambda_{p}=\lambda_{1}^{\prime}\left(-L, \mathbb{R}^{N}\right),
$$

as it is seen by taking $\phi=\varphi_{p}$ in (1.8). But, in general, we do not have equality. As an example, for the operator $L u=u^{\prime \prime}-u^{\prime}$, which is periodic with any positive period, one has $\lambda_{p}=0$, while $\lambda_{R}=1 / 4+\pi^{2} /\left(4 R^{2}\right) \rightarrow 1 / 4=\lambda_{1}(-L, \mathbb{R})$ as $R \rightarrow+\infty$ (see the arguments in the proof of Theorem 6.8 below).

However, note that the fact that the operator is non-self adjoint does not garantee in general that $\lambda_{1}\left(-L, \mathbb{R}^{N}\right)>\lambda_{p}$. Indeed, for an operator of the type $L u=u^{\prime \prime}+q(x) u^{\prime}$, where $q \in C^{0, \gamma}(\mathbb{R})$ is periodic with zero average, then $\lambda_{1}\left(-L, \mathbb{R}^{N}\right)=\lambda_{p}$. This result is actually a particular case of the following theorem, which generalizes Proposition 6.6 to non-self adjoint operators with gradient-type drift.

Theorem 6.8 Consider the operator

$$
-L u:=-\partial_{i}\left(a_{i j}(x) \partial_{j} u\right)-q_{i}(x) \partial_{i} u-c(x) u, \quad x \in \mathbb{R}^{N},
$$

where $a_{i j}, q_{i}$, c are periodic in $x$, with the same period $\left(l_{1}, \cdots, l_{N}\right)$, the matrix field $A(x)=$ $\left(a_{i j}(x)\right)_{1 \leq i, j \leq N}$ is in $C^{1, \gamma}\left(\mathbb{R}^{N}\right)$, elliptic and symmetric, the vector field $q=\left(q_{1}, \cdots, q_{N}\right) \in$ $C^{1, \gamma}\left(\mathbb{R}^{N}\right)$ and $c \in C^{0, \gamma}\left(\mathbb{R}^{N}\right)$. Assume that there is a function $Q \in C^{2, \gamma}\left(\mathbb{R}^{N}\right)$ such that $a_{i j} \partial_{j} Q=q_{i}$ for all $i=1, \ldots, N$ and assume that the vector field $A^{-1} q$ has zero average in the periodicity cell $C=\left(0, l_{1}\right) \times \cdots \times\left(0, l_{N}\right)$. Then $\lambda_{1}\left(-L, \mathbb{R}^{N}\right)=\lambda_{p}=\lambda_{1}^{\prime}\left(-L, \mathbb{R}^{N}\right)$, where $\lambda_{p}$ is the periodic principal eigenvalue of $-L$ in $\mathbb{R}^{N}$.

Proof. We show preliminarily that $Q$ is periodic with period $\left(l_{1}, \cdots, l_{N}\right)$. For $i=1, \cdots, N$ call $Q^{i}(x):=Q\left(x+l_{i} e_{i}\right)-Q(x)$. The functions $Q^{i}$ are constant because

$$
\nabla Q^{i}(x)=\nabla Q\left(x+l_{i} e_{i}\right)-\nabla Q(x)=\left(A^{-1} q\right)\left(x+l_{i} e_{i}\right)-\left(A^{-1} q\right)(x)=0
$$

(note that $A^{-1}$ has the same periodicity as $A$ ). Call $k^{i}$ the constant value of $Q^{i}$; we have:

$$
k^{i}\left|l_{1}\right| \cdots\left|l_{N}\right|=\int_{C} Q^{i}(x) d x=\int_{C} d x \int_{0}^{l_{i}} \partial_{i} Q\left(x+t e_{i}\right) d t=\int_{0}^{l_{i}} d t \int_{C}\left(A^{-1} q\right)_{i}\left(x+t e_{i}\right) d x=0 .
$$

Hence the $Q^{i}$ are identically equal to zero and then $Q$ is periodic. 
For $R>0$ call $\lambda_{R}$ and $\varphi_{R}$ the principal eigenvalue and, respectively, the principal eigenfunction of $-L$ in $B_{R}$, with Dirichlet boundary conditions. For $x \in \overline{B_{R}}$, define $\tilde{\varphi}_{R}(x):=\varphi_{R}(x) e^{Q(x) / 2}$. Direct computation shows that the function $\tilde{\varphi}_{R}$, which is positive in $B_{R}$ and vanishes on $\partial B_{R}$, satisfies $\left(\tilde{L}+\lambda_{R}\right) \tilde{\varphi}_{R}=0$ in $B_{R}$, where

$$
-\tilde{L} u:=-\partial_{i}\left(a_{i j}(x) \partial_{j} u\right)-\left(-\frac{1}{2} \partial_{i}\left(a_{i j}(x) \partial_{j} Q\right)-\frac{1}{4} a_{i j}(x) \partial_{i} Q \partial_{j} Q+c(x)\right) u .
$$

This means that $\lambda_{R}$ is the principal eigenvalue in $B_{R}$ of $-\tilde{L}$ too and then, by Proposition 4.2 , $\lambda_{1}\left(-L, \mathbb{R}^{N}\right)=\lim _{R \rightarrow \infty} \lambda_{R}=\lambda_{1}\left(-\tilde{L}, \mathbb{R}^{N}\right)$. But the operator $\tilde{L}$ is self adjoint and periodic. Applying Proposition 6.6 to $\tilde{L}$ we find that $\lambda_{1}\left(-L, \mathbb{R}^{N}\right)$ is equal to the periodic principal eigenvalue of $-\tilde{L}$ in $\mathbb{R}^{N}$. Call $\tilde{\varphi}_{p}$ the associated periodic principal eigenfunction, namely $\tilde{\varphi}_{p}$ is periodic in $x$, positive and satisfies $\left(\tilde{L}+\lambda_{1}\left(-L, \mathbb{R}^{N}\right)\right) \tilde{\varphi}_{p}=0$ in $\mathbb{R}^{N}$. Again with the same calculations, we find that the function $\psi(x):=\tilde{\varphi}_{p}(x) e^{-Q(x) / 2}$ satisfies: $\left(L+\lambda_{1}\left(-L, \mathbb{R}^{N}\right)\right) \psi=0$ in $\mathbb{R}^{N}$ and it is periodic in $x$ because of the periodicity of $Q$. Consequently $\psi$ coincides with the periodic principal eigenfunction of $-L$ in $\mathbb{R}^{N}$ and then $\lambda_{1}\left(-L, \mathbb{R}^{N}\right)=\lambda_{p}\left(=\lambda_{1}^{\prime}\left(-L, \mathbb{R}^{N}\right)\right.$, by Proposition 6.3).

We show now that, in the one dimensional self adjoint case, $\lambda_{1}(-L, \mathbb{R}) \leq \lambda_{1}^{\prime}(-L, \mathbb{R})$, without periodicity assumption. To do that we use the following result.

Lemma 6.9 Let $g: \mathbb{R}^{N} \rightarrow \mathbb{R}$ be a nonnegative, not identically equal to zero function in $L^{\infty}\left(\mathbb{R}^{N}\right)$ and call $C_{R}:=B_{R} \backslash B_{R-1}$.

(i) We have that

$$
\liminf _{R \rightarrow \infty} \frac{\int_{C_{R}} g}{\int_{B_{R-1}} g}=0 .
$$

(ii) If $N=1$ then

$$
\lim _{R \rightarrow \infty} \frac{\int_{C_{R}} g}{\int_{B_{R-1}} g^{2}}=0 .
$$

Proof. To prove (i) suppose, by a contradiction, that

$$
\liminf _{R \rightarrow \infty} \frac{\int_{C_{R}} g}{\int_{B_{R-1}} g}>0 .
$$

Hence there exist $\varepsilon>0$ and $R_{\varepsilon}>1$ such that

$$
\forall R \geq R_{\varepsilon}, \quad \frac{\int_{C_{R}} g}{\int_{B_{R-1}} g} \geq \varepsilon>0 .
$$


Consider the sequence $\left(R_{n}\right)_{n \geq 0}$ defined by $R_{n}:=R_{\varepsilon}+n$, and set $a_{n}:=\int_{C_{R_{n}}} g$. For $n \in \mathbb{N}$ we have that $B_{R_{n}} \supset \bigcup_{k=0}^{n} C_{R_{k}}$ and then $a_{n} \geq \varepsilon \sum_{k=0}^{n-1} a_{k}$. It is easy to check that the last inequality implies:

$$
\forall n \geq 1, \quad a_{n} \geq \varepsilon a_{0}(1+\varepsilon)^{n-1} .
$$

On the other hand we have that

$$
a_{n}=\int_{C_{R_{n}}} g \leq\|g\|_{L^{\infty}\left(\mathbb{R}^{N}\right)} K R_{n}^{N-1} \leq K^{\prime} n^{N-1},
$$

where $K, K^{\prime}$ are two positive constants. This is in contradiction with (6.7).

The proof of (ii) is similar: suppose by a contradiction that there exist $\varepsilon>0$ and a sequence $\left(R_{n}\right)_{n \in \mathbb{N}}$ of numbers greater than one such that: $R_{n} \rightarrow+\infty$ and

$$
\forall n \in \mathbb{N}, \quad \frac{\int_{C_{R_{n}}} g}{\int_{B_{R_{n}-1}} g^{2}} \geq \varepsilon>0 .
$$

We can assume, without loss of generality, that

$$
\forall n \in \mathbb{N}, \quad R_{n+1} \geq R_{n}+1 .
$$

By (6.8) it follows that $C_{R_{k}} \subset B_{R_{n}-1}$ for $k \leq n-1$, and also that $C_{R_{k}} \cap C_{R_{h}}=\emptyset$ if $k \neq h$. Setting $a_{n}:=\left(\int_{C_{R_{n}}} g^{2}\right)^{1 / 2}$ for $n \in \mathbb{N}$ we find, by Cauchy-Schwarz inequality:

$$
a_{n}=\left(\int_{C_{R_{n}}} g^{2}\right)^{1 / 2} \geq \frac{1}{\sqrt{2}} \int_{C_{R_{n}}} g \geq \frac{\varepsilon}{\sqrt{2}} \int_{B_{R_{n}-1}} g^{2} \geq \frac{\varepsilon}{\sqrt{2}} \sum_{k=1}^{n-1} \int_{C_{R_{k}}} g^{2}=\frac{\varepsilon}{\sqrt{2}} \sum_{k=1}^{n-1} a_{k}^{2} .
$$

Therefore $a_{n} \geq \varepsilon a_{1}^{2} / \sqrt{2}$ for $n \geq 2$ and then

$$
a_{n} \geq \frac{\varepsilon^{3} a_{1}^{4}(n-2)}{2 \sqrt{2}}
$$

which goes to plus infinity as $n$ goes to infinity. This is a contradiction because

$$
a_{n}=\left(\int_{C_{R_{n}}} g^{2}\right)^{1 / 2} \leq \sqrt{2}\|g\|_{L^{\infty}(\mathbb{R})} .
$$

Remark 6.10 Statement (ii) of Lemma 6.9 does not hold if $N \geq 3$, as it is shown by choosing the function

$$
g(x)= \begin{cases}1 & \text { if } x \in B_{1} \\ |x|^{3 / 2-N} & \text { otherwise }\end{cases}
$$


Proposition 6.11 Consider the one dimensional, self adjoint operator

$$
-L u=-\left(a(x) u^{\prime}\right)^{\prime}-c(x) u, \quad x \in \mathbb{R},
$$

with $c \in C_{\text {loc }}^{0, \gamma}(\mathbb{R}) \cap L^{\infty}(\mathbb{R}), a \in C_{\text {loc }}^{1, \gamma}(\mathbb{R}) \cap W^{1, \infty}(\mathbb{R})$ such that $0<\alpha \leq a(x)$ for all $x \in \mathbb{R}$. Then $\lambda_{1}(-L, \mathbb{R}) \leq \lambda_{1}^{\prime}(-L, \mathbb{R})$.

Proof. The proof is similar to the one of Proposition 6.6. Suppose by a contradiction that $\lambda_{1}(-L, \mathbb{R})>\lambda_{1}^{\prime}(-L, \mathbb{R})$. There exist then $\lambda<\lambda_{1}(-L, \mathbb{R})$ and a positive bounded function $\phi \in C^{2}(\mathbb{R}) \cap W^{1, \infty}(\mathbb{R})$ such that $-(L+\lambda) \phi \leq 0$. Consider the same family of functions $\left(\chi_{R}\right)_{R>2}$ as in the proof of Proposition 6.6, with the additional assumption: $\chi_{R} \leq 1$ in $\mathbb{R}$, for any $R>0$. Fix $R>0$ and call $\lambda_{R}:=\lambda_{1}(-L,(-R, R))$. Using the variational representation for $\lambda_{R}$ we obtain:

$$
\begin{aligned}
\lambda_{R} & \leq \frac{\int_{-R}^{R}\left[a(x)\left(\left(\phi \chi_{R}\right)^{\prime}\right)^{2}-c(x)\left(\phi \chi_{R}\right)^{2}\right]}{\int_{-R}^{R}\left(\phi \chi_{R}\right)^{2}} \\
& \leq \lambda \frac{\int_{-R+1}^{R-1} \phi^{2}}{\int_{-R}^{R}\left(\phi \chi_{R}\right)^{2}}-\frac{\int_{C_{R}}\left[\left(a(x)\left(\phi \chi_{R}\right)^{\prime}\right)^{\prime} \phi \chi_{R}+c(x)\left(\phi \chi_{R}\right)^{2}\right]}{\int_{-R}^{R}\left(\phi \chi_{R}\right)^{2}} \\
& \leq \lambda\left(\eta_{R}\right)^{-1}+K_{1} \frac{\int_{C_{R}}^{R} \phi \chi_{R}}{\int_{-R}^{R}\left(\phi \chi_{R}\right)^{2}}+K_{2} \frac{\int_{C_{R}}^{R}\left(\phi \chi_{R}\right)^{2}}{\int_{C_{R}}^{R}\left(\phi \chi_{R}\right)^{2}} \\
& \leq \lambda\left(\eta_{R}\right)^{-1}+K_{1} \frac{\int_{C_{R}}^{\phi^{2}}}{\int_{-R+1}^{R-1} \phi^{2}}+K_{2} \frac{\int_{-R+1}^{R-1} \phi^{2}}{\int^{R+1}}
\end{aligned}
$$

where $K_{1}$ and $K_{2}$ are two positive constants which do not depend on $R$, and

$$
1 \leq \eta_{R}=1+\frac{\int_{C_{R}}\left(\phi \chi_{R}\right)^{2}}{\int_{B_{R-1}} \phi^{2}} \leq 1+\frac{\int_{C_{R}} \phi^{2}}{\int_{-R+1}^{R-1} \phi^{2}} .
$$

Thus, using Lemma 6.9 part (i) (with $g=\phi^{2}$ ) and part (ii) (with $g=\phi$ ), it turns out that $\liminf _{R \rightarrow \infty} \lambda_{R} \leq \liminf _{R \rightarrow \infty} \lambda\left(\eta_{R}\right)^{-1}$. If $\lambda$ is nonnegative then $\lambda\left(\eta_{R}\right)^{-1} \leq \lambda$, else

$$
\liminf _{R \rightarrow \infty} \lambda\left(\eta_{R}\right)^{-1}=\lambda\left(\liminf _{R \rightarrow \infty} \eta_{R}\right)^{-1} \leq \lambda\left(1+\liminf _{R \rightarrow \infty} \frac{\int_{C_{R}} \phi^{2}}{\int_{-R+1}^{R-1} \phi^{2}}\right)^{-1}=\lambda
$$


again by Lemma 6.9 part (i). In both case we find: $\liminf _{R \rightarrow \infty} \lambda_{R} \leq \lambda<\lambda_{1}(-L, \mathbb{R})$, which is a contradiction because, by Proposition 4.2 , we know that $\lambda_{1}(-L, \mathbb{R})=\lim _{R \rightarrow \infty} \lambda_{R}$.

Remark 6.12 Let $\varphi_{\infty}$ be a generalized principal eigenfunction in $\mathbb{R}^{N}$ of an elliptic operator $-L$ (constructed, for example, as in the proof of Proposition 4.2), i.e. $\varphi_{\infty}>0$ and $(L+$ $\left.\lambda_{1}\left(-L, \mathbb{R}^{N}\right)\right) \varphi_{\infty}=0$ in $\mathbb{R}^{N}$. We claim that, if $\inf _{\mathbb{R}^{N}} \varphi_{\infty}>0$, then $\lambda_{1}^{\prime}\left(-L, \mathbb{R}^{N}\right) \geq \lambda_{1}\left(-L, \mathbb{R}^{N}\right)$. Consequently, if $L$ is periodic, a sufficient condition in order to have $\lambda_{1}\left(-L, \mathbb{R}^{N}\right)=\lambda_{p}$ in $\mathbb{R}^{N}$ is: $\inf _{\mathbb{R}^{N}} \varphi_{\infty}>0$. The second assertion follows from the first one because, by Proposition $6.3, \lambda_{p}=\lambda_{1}^{\prime}\left(-L, \mathbb{R}^{N}\right)$, and, as already emphasized, the inequality $\lambda_{1}\left(-L, \mathbb{R}^{N}\right) \geq \lambda_{p}$ is an immediate consequence of the definition (1.8). In order to prove the first statement suppose, by a contradiction, that $\lambda_{1}\left(-L, \mathbb{R}^{N}\right)>\lambda_{1}^{\prime}\left(-L, \mathbb{R}^{N}\right)$. There exists then a number $\lambda<\lambda_{1}\left(-L, \mathbb{R}^{N}\right)$ and positive bounded function $\phi$ such that $-(L+\lambda) \phi \leq 0$ in $\mathbb{R}^{N}$. Chose $\varepsilon>0$ small enough in order to have:

$$
-(L+\lambda) \varphi_{\infty}=\left(\lambda_{1}\left(-L, \mathbb{R}^{N}\right)-\lambda\right) \varphi_{\infty} \geq \varepsilon, \quad \text { in } \mathbb{R}^{N} .
$$

Applying part (ii) of Lemma 2.1 to the operator $\mathcal{L}=L+\lambda$ we find a contradiction with the existence of $\phi$.

\section{The evolution equation and large time behavior}

Theorem 7.1 Let $u(t, x)$ be the solution of (1.10), with an initial datum $u_{0}$ which is uniformly continuous, nonnegative bounded and not identically equal to 0.

1) Under assumptions (1.4), (1.5) and (1.6) the function $u(t, x)$ converges in $C_{\text {loc }}^{2}\left(\mathbb{R}^{N}\right)$, as $t \rightarrow+\infty$, to the unique positive bounded solution of (1.1). Furthermore the same conclusion holds under assumptions (1.4), (1.5) and (1.9), provided that the principal eigenvalue in $\mathbb{R}^{N}$ of any limit operator associated with $-\mathcal{L}_{0}$ is negative.

2) If (1.4) and (6.2) hold and if the quantity $\lambda_{1}^{\prime}\left(-\mathcal{L}_{0}, \mathbb{R}^{N}\right)$, given by (6.1), is positive, then $u(t, x)$ converges to 0 in $C_{\text {loc }}^{2}\left(\mathbb{R}^{N}\right)$ as $t \rightarrow+\infty$.

Proof. Let us first prove part 2). Consider the solution $\bar{v}$ of (1.10) with

$$
\bar{v}(0, x)=\max \left\{M,\left\|u_{0}\right\|_{L^{\infty}\left(\mathbb{R}^{N}\right)}\right\},
$$

where $M$ is the constant in (1.4). Since $\bar{v}(0, x)$ is a super-solution of the stationary problem (1.1), it follows from the parabolic maximum principle that $\bar{v}$ is nonincreasing in $t$. Furthermore, parabolic estimates imply that, as $t$ goes to infinity, $\bar{v}(t, x)$ converges in $C_{l o c}^{2}\left(\mathbb{R}^{N}\right)$ to a function $p$ which is a nonnegative bounded solution of (1.1). Since $\lambda_{1}^{\prime}\left(-\mathcal{L}_{0}, \mathbb{R}^{N}\right)>0$ we conclude from Proposition 6.1 that $p \equiv 0$. Using the parabolic maximum principle it turns out that $0 \leq u(t, x) \leq \bar{v}(t, x)$, for all $(t, x) \in \mathbb{R}_{+} \times \mathbb{R}^{N}$, and then statement 2$)$ is proved.

Let us now prove part 1$)$. Under assumptions which are made, it follows from Theorem 1.1 or Theorem 1.3 that there exists a unique positive bounded solution $p$ of (1.1). Furthermore, in case of assumptions (1.4), (1.5), (1.9) and that $\lambda_{1}\left(-\mathcal{L}^{*}, \mathbb{R}^{N}\right)<0$ for some limit operator $-\mathcal{L}^{*}$, Proposition 5.3 yields $\lambda_{1}\left(-\mathcal{L}_{0}, \mathbb{R}^{N}\right)<0$. On the other hand, under assumptions 
(1.4), (1.5) and (1.6), the estimates (4.2) in Theorem 4.4 with $\Omega=\mathbb{R}^{N}$ implies again: $\lambda_{1}\left(-\mathcal{L}_{0}, \mathbb{R}^{N}\right)<0$.

From Theorem 5.1, and with the same notations as there, there exists $R>0$ large enough such that the principal eigenvalue $\lambda_{R}$ of $-\mathcal{L}_{0}$ in $B_{R}$ with Dirichlet boundary conditions is negative. Therefore, denoting $\varphi_{R}$ a corresponding principal eigenfunction, there exists $\varepsilon>0$ small enough such that the function

$$
v(x)= \begin{cases}\varepsilon \varphi_{R}(x) & \text { if } x \in B_{R} \\ 0 & \text { if } x \notin B_{R}\end{cases}
$$

is a subsolution of (1.1). Even if it means decreasing $\varepsilon$, one can assume that $v(x) \leq u(1, x)$ in $\mathbb{R}^{N}$ (indeed, $u(1, \cdot)$ is continuous in $\mathbb{R}^{N}$ and positive from the strong parabolic maximum principle). Therefore, $u(t+1, x) \geq \underline{v}(t, x)$ for all $t \geq 0$ and $x \in \mathbb{R}^{N}$, where $\underline{v}$ solves (1.10) with initial datum $v$. Furthermore, $\underline{v}$ is nondecreasing in time $t$. On the other hand, as in the proof of part 2) above, one has $0 \leq u(t, x) \leq \bar{v}(t, x)$ for all $t \geq 0$ and $x \in \mathbb{R}^{N}$, and $\bar{v}(t, x) \searrow \bar{p}$ in $C_{\text {loc }}^{2}\left(\mathbb{R}^{N}\right)$ as $t \rightarrow+\infty$, where $\bar{p}$ is a bounded nonnegative solution of (1.1). Therefore, $\underline{v}(t, x) \nearrow \underline{p}$ in $C_{l o c}^{2}\left(\mathbb{R}^{N}\right)$ as $t \rightarrow+\infty$, where $\underline{p}$ is a bounded nonnegative solution of (1.1) such that $0 \leq v(x) \leq p(x) \leq \bar{p}(x)$ in $\mathbb{R}^{N}$. Therefore, the strong maximum principle implies that $p$ and $\bar{p}$ are positive (and bounded) solutions of (1.1), and they are then equal to the unique such solution, $p$. One concludes that $u(t, x) \rightarrow p(x)$ in $C_{l o c}^{2}\left(\mathbb{R}^{N}\right)$ as $t \rightarrow+\infty$.

\section{Appendix: Construction of a nonnegative subsolu- tion}

To prove Lemma 3.1, we first build a subsolution in $\mathbb{R}$ (see next lemma), then we rotate it in order to obtain a function of $N$ variables.

Lemma 8.1 Let $\beta, \eta$ and $\theta$ be three arbitrary positive real numbers. Then there exist a nonnegative function $h \in C^{2}(\mathbb{R} ; \mathbb{R})$ and a positive number $t$ such that:

$$
\begin{array}{rll}
h(\rho)=0 & \text { for } & \rho \leq 0, \\
h^{\prime}(\rho)>0 & \text { for } & 0<\rho<t, \\
h(\rho)=\text { constant } & \text { for } & \rho \geq t,
\end{array}
$$

and

$$
\forall(\rho, x) \in(0, t] \times \mathcal{A}, \quad-\mathcal{L}_{A, Q, C} h(\rho, x):=-A(x) h^{\prime \prime}(\rho)+Q(x) h^{\prime}(\rho)-C(x) h(\rho)<0,
$$

for any nonnegative functions $A, Q, C$ and subset $\mathcal{A}$ of $\mathbb{R}^{N}$, verifying

$$
\forall x \in \mathcal{A}, \quad A(x) \leq \beta, \quad Q(x) \leq \eta \quad \text { and } \quad 4 A(x) C(x)-Q^{2}(x) \geq \theta .
$$

Proof. We will prove this lemma using a constructive method. Set

$$
h(\rho)=\left\{\begin{array}{cl}
0 & \text { for } \rho \leq 0 \\
\rho^{n} & \text { for } \rho \in(0, r]
\end{array}\right.
$$


where the integer $n \geq 3$ and the real $r>0$ are to be chosen. Let $A, Q$ and $C$ be three nonnegative functions, defined on a generic set $\mathcal{A}$, satisfying (8.1). Note that, by the last inequality in (8.1), the functions $A$ and $C$ are strictly positive. For $(\rho, x) \in(0, r] \times \mathcal{A}$ we have:

$$
\begin{aligned}
-\mathcal{L}_{A, Q, C} h(\rho, x) & =\left[A(x)\left(n-n^{2}\right)+Q(x) n \rho-C(x) \rho^{2}\right] \rho^{n-2} \\
& \leq\left[A(x)\left(n-n^{2}\right)+\frac{[Q(x)]^{2}}{4 C(x)} n^{2}\right] \rho^{n-2} \\
& =\left(A(x)-\frac{4 A(x) C(x)-[Q(x)]^{2}}{4 C(x)} n\right) n \rho^{n-2} .
\end{aligned}
$$

Using (8.1), one sees that, if $A(x) C(x)>\eta^{2}\left(\geq Q(x)^{2}\right)$, the last quantity is less than

$$
\left(A(x)-\frac{4 A(x) C(x)-A(x) C(x)}{4 C(x)} n\right) n \rho^{n-2}=\left(1-\frac{3 n}{4}\right) A(x) n \rho^{n-2}<0,
$$

else it is less than or equal to

$$
\left(1-\frac{\theta}{4 \eta^{2}} n\right) A(x) n \rho^{n-2}
$$

Thus, $n$ (in $\mathbb{N}$ ) may be chosen large enough (depending only on $\eta$ and $\theta$ ) in order to have: $-\mathcal{L}_{A, Q, C} h<0$ in $(0, r] \times \mathcal{A}$.

Take now $r>0$ such that

$$
\left(\eta n-\frac{\theta}{4 \beta} r\right) r^{n-1} \leq-\beta-1
$$

and

$$
h^{\prime \prime}(r)=n(n-1) r^{n-2}>1 .
$$

For $\rho \in(r, s]$ we set $h^{\prime \prime}(\rho)=h^{\prime \prime}(r)-\gamma(\rho-r)$, where $s$ and $\gamma$ are two positive constants that will be chosen later. Imposing $h^{\prime \prime}(s)=-1$ one finds that $s=r+\frac{h^{\prime \prime}(r)+1}{\gamma}$. Since $h^{\prime \prime}(\rho)>0$ for $\rho \in\left[r, r+\frac{h^{\prime \prime}(r)}{\gamma}\right)$ and $h^{\prime \prime}(\rho)<0$ for $\rho \in\left(r+\frac{h^{\prime \prime}(r)}{\gamma}, s\right]$, we have that

$$
\forall \rho \in[r, s], \quad h^{\prime}(\rho) \leq h^{\prime}\left(r+\frac{h^{\prime \prime}(r)}{\gamma}\right)=h^{\prime}(r)+\frac{\left[h^{\prime \prime}(r)\right]^{2}}{2 \gamma} .
$$

Furthermore $h^{\prime}(s)=h^{\prime}(r)+\frac{h^{\prime \prime}(r)+1}{2 \gamma}\left(h^{\prime \prime}(r)-1\right)>h^{\prime}(r)$ by $(8.3)$ and so the concavity of $h^{\prime}$ in the interval $[r, s]$ implies: $h^{\prime} \geq h^{\prime}(r)>0$ in $[r, s]$. Thus, $h \geq h(r)$ in $[r, s]$. Using this and the fact that $4 A(x) C(x) \geq \theta+Q^{2}(x) \geq \theta$ one gets, for $(\rho, x) \in(r, s] \times \mathcal{A}$,

$$
\begin{aligned}
-\mathcal{L}_{A, Q, C} h(\rho, x) & \leq \beta+\eta\left(h^{\prime}(r)+\frac{\left[h^{\prime \prime}(r)\right]^{2}}{2 \gamma}\right)-\frac{\theta}{4 A(x)} h(r) \\
& \leq \beta+\left(\eta n-\frac{\theta}{4 \beta} r\right) r^{n-1}+\frac{\eta}{2 \gamma}\left[h^{\prime \prime}(r)\right]^{2}
\end{aligned}
$$


So, by $(8.2),-\mathcal{L}_{A, Q, C} h \leq-1+\frac{\eta}{2 \gamma}\left[h^{\prime \prime}(r)\right]^{2}$ in $(r, s] \times \mathcal{A}$, and then it is sufficient to take $\gamma>0$ sufficiently large to obtain:

$$
-\mathcal{L}_{A, Q, C} h(\rho, x)<0 \quad \text { in }(r, s] \times \mathcal{A} .
$$

For $\rho \in(s, t]$ we finally set $h^{\prime \prime}(\rho)=-1+\delta(\rho-s)$, where the constants $t$ and $\delta$ are the solutions of the system

$$
\left\{\begin{array}{l}
h^{\prime}(t)=0 \\
h^{\prime \prime}(t)=0
\end{array}\right.
$$

Direct computation leads to:

$$
\delta=\frac{1}{2 h^{\prime}(s)}, \quad t=s+2 h^{\prime}(s)
$$

Since $h^{\prime \prime}<0$ in $(s, t)$ we see that $h^{\prime}$ is decreasing and positive in $(s, t)$, and then $h$ is increasing in $(s, t)$. Hence, we can conclude that, for $(\rho, x) \in(s, t] \times \mathcal{A}$,

$$
\begin{aligned}
-\mathcal{L}_{A, Q, C} h(\rho, x) & =-A(x) h^{\prime \prime}(\rho)+Q(x) h^{\prime}(\rho)-C(x) h(\rho) \\
& \leq-A(x) h^{\prime \prime}(s)+Q(x) h^{\prime}(s)-C(x) h(s) \\
& =-\mathcal{L}_{A, Q, C} h(s, x) \\
& <0 .
\end{aligned}
$$

Clearly $h \in C^{2}(\mathbb{R})$. Let us underline the fact that $h$ does not depend on $A, Q$ and $C$, but only on $\beta, \eta$ and $\theta$.

Now we are ready to prove Lemma 3.1.

Proof of Lemma 3.1. By (3.1) we have that, for $s>0$,

$$
\inf _{x \in \mathcal{A}}\left[4 \alpha(x) c(x)-\left(|q(x)|+\frac{N \beta}{s}\right)^{2}\right] \geq b-\frac{2 a N \beta}{s}-\left(\frac{N \beta}{s}\right)^{2} .
$$

Hence, $s>0$ may be chosen large enough (depending only on $a, b, \beta$ and $N$ ) in order to have:

$$
\theta:=\inf _{x \in \mathcal{A}}\left[4 \alpha(x) c(x)-\left(|q(x)|+\frac{N \beta}{s}\right)^{2}\right]>0 .
$$

Set

$$
\eta:=a+\frac{N \beta}{s}
$$

and consider $h$ and $t$ given by Lemma 8.1, associated with the positive constants $\eta, \theta$ and $\beta$. Set $r=s+t$ and define the function

$$
\psi(x):=h(r-|x|), \quad x \in \mathbb{R}^{N} .
$$

The function $\psi$ is $C^{2}$ on all $\mathbb{R}^{N}$ (observe especially that it is constant in $B_{s}$ ). Moreover it is strictly positive in $B_{r}$ and it is identically equal to zero in $\mathbb{R}^{N} \backslash B_{r}$. 
Suppose that there exists $x_{0} \in \mathcal{A}$ such that $B_{r}\left(x_{0}\right) \subset \mathcal{A}$. For $x \in B_{s}\left(x_{0}\right)$ we have that $\tau_{x_{0}} \psi$ is constantly equal to $h(t)$ and then $-L \tau_{x_{0}} \psi(x)=-c(x) h(t)<0$, because $c>0$ in $\mathcal{A}$ by (3.1). For $x \in B_{r}\left(x_{0}\right) \backslash B_{s}\left(x_{0}\right)$, direct computation leads to:

$$
\begin{aligned}
-L \tau_{x_{0}} \psi(x)= & -\frac{a_{i j}(x)\left(x-x_{0}\right)_{i}\left(x-x_{0}\right)_{j}}{\left|x-x_{0}\right|^{2}} h^{\prime \prime}\left(r-\left|x-x_{0}\right|\right) \\
& +\left(\frac{a_{i i}(x)}{\left|x-x_{0}\right|}-\frac{a_{i j}(x)\left(x-x_{0}\right)_{i}\left(x-x_{0}\right)_{j}}{\left|x-x_{0}\right|^{3}}\right) h^{\prime}\left(r-\left|x-x_{0}\right|\right) \\
& -q(x) \cdot \frac{x-x_{0}}{\left|x-x_{0}\right|} h^{\prime}\left(r-\left|x-x_{0}\right|\right)-c(x) h\left(r-\left|x-x_{0}\right|\right)
\end{aligned}
$$

and then

$$
\begin{aligned}
-L \tau_{x_{0}} \psi(x) \leq & -\frac{a_{i j}(x)\left(x-x_{0}\right)_{i}\left(x-x_{0}\right)_{j}}{\left|x-x_{0}\right|^{2}} h^{\prime \prime}\left(r-\left|x-x_{0}\right|\right) \\
& +\left(|q(x)|+\frac{N \beta}{s}\right) h^{\prime}\left(r-\left|x-x_{0}\right|\right) \\
& -c(x) h\left(r-\left|x-x_{0}\right|\right) .
\end{aligned}
$$

For $x \in \mathcal{A}$, denote

$$
A(x):=\frac{a_{i j}(x)\left(x-x_{0}\right)_{i}\left(x-x_{0}\right)_{j}}{\left|x-x_{0}\right|^{2}}, \quad Q(x):=|q(x)|+\frac{N \beta}{s} \quad \text { and } \quad C(x):=c(x) .
$$

By (3.1) and (8.5) we have that $Q(x) \leq \eta$ for all $x \in \mathcal{A}$. Furthermore, since $C$ is positive, the ellipticity of the $a_{i j}$ and (8.4) yield:

$$
\forall x \in \mathcal{A}, \quad 4 A(x) C(x)-Q(x)^{2} \geq 4 \alpha(x) C(x)-Q(x)^{2} \geq \theta .
$$

Consequently, the functions $A, Q$ and $C$ satisfy (8.1) and then it follows that $-L \tau_{x_{0}} \psi<0$ also in $B_{r}\left(x_{0}\right) \backslash B_{s}\left(x_{0}\right)$.

\section{References}

[1] H. Berestycki. Le nombre de solutions de certains problèmes semi-linéaires elliptiques. J. Func. Anal. 40 (1981), pp 1-29.

[2] H. Berestycki, O. Dickmann, K. Nagelkerke and P. Zegeling. Can a species keep pace with a shiftering? preprint.

[3] H. Berestycki, F. Hamel, and N. Nadirashvili. The speed of propagation for KPP type problems. I : Periodic framework. J. Eur. Math. Soc. 7 (2005), pp 173-213.

[4] H. Berestycki, F. Hamel, and L. Roques. Analysis of the periodically fragmented environment model : I - Influence of periodic heterogeneous environment on species persistence. J. Math. Biology 51 (2005), pp 75-113.

[5] H. Berestycki and P.-L. Lions. Some applications of the method of super- and subsolutions. Bifurcation and Nonlinear Eigenvalue Problems, (Bardos, Lasry \& Schatzman, eds.). Lecture Notes in Math 782 (1980), Springer-Verlag, New York, pp 16-41. 
[6] H. Berestycki and L. Nirenberg. Travelling fronts in cylinders. Ann. Inst. H. Poincaré Anal. Non Linéaire 9 (1992), pp 497-572.

[7] H. Berestycki, L. Nirenberg, and S. R. S. Varadhan. The principal eigenvalue and maximum principle for second-order elliptic operators in general domains. Comm. Pure Appl. Math. 47 (1994), pp 47-92.

[8] S. Bochner. A new approach to almost periodicity. Proc. Nat. Ac. Sci. USA 48 (1963), pp 2039-2043.

[9] J. Engländer and A. E. Kyprianou. Local extinction versus local exponential growth for spatial branching processes. Ann. Probab. 32 (2004), pp 78-99.

[10] J. Engländer and R. G. Pinsky. On the construction and support properties of measurevalued diffusions on $D \subseteq R^{d}$ with spatially dependent branching. Ann. Probab. 27 (1999), pp 684-730.

[11] D. Gilbarg and N. S. Trudinger. Elliptic partial differential equations of second order. Springer-Verlag, Berlin, second edition, 1983.

[12] A. N. Kolmogorov, I. G. Petrovskiŭ, and N. S. Piskunov. Étude de l'équation de la diffusion avec croissance de la quantité de matière et son application à un problème biologique. Bulletin Université d'Etat à Moscou (Bjul. Moskowskogo Gos. Univ.), Série internationale A 1 (1937), pp 1-26.

[13] R. G. Pinsky. Transience, recurrence and local extinction properties of the support for supercritical finite measure-valued diffusions. Ann. Probab. 24 (1996), pp 237-267.

[14] M. H. Protter and H. F. Weinberger. Maximum principles in differential equations. Prentice-Hall Inc., Englewood Cliffs, N.J., 1967. 